\title{
Hydrazulene Ring Systems via Heteroatom-Assisted [1,2]-Shift of Oxonium and Sulfonium Ylides
}

\author{
Graham K. Murphy and F. G. West \\ Department of Chemistry, University of Alberta, Edmonton, $A B$, \\ Canada T6G 2 G2 \\ frederick.west@ualberta.ca
}

\section{Supporting Information}

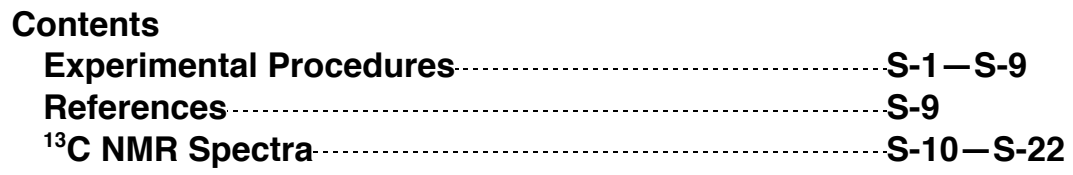

General. Reactions were carried out in flame-dried glassware under a positive nitrogen atmosphere unless otherwise stated. Transfer of anhydrous solvents and reagents was accomplished with oven-dried syringes or cannula. Solvents were distilled before use: methylene chloride from calcium hydride, tetrahydrofuran and diethylether from sodium/benzophenone ketyl, toluene from sodium metal. Ethereal diazomethane (hazard!) was prepared from Diazald according to literature procedures. ${ }^{i}$ Thin layer chromatography was performed on glass plates precoated with $0.25 \mathrm{~mm}$ Kieselgel $60 \mathrm{~F}_{254}$ (Merck). Flash chromatography columns were packed with 230-400 mesh silica gel (Merck). Radial chromatography was carried out on a Chromatotron 7924T (Harrison Research) with plates prepared using silica gel $60 \mathrm{~F}_{254}$ with gypsum binder (EM) on glass rotors. Proton nuclear magnetic resonance spectra $\left({ }^{1} \mathrm{H}\right.$ NMR) were recorded at $400 \mathrm{MHz}$ or $500 \mathrm{MHz}$ and coupling constants $(J)$ are reported in Hertz $(\mathrm{Hz})$. Carbon nuclear magnetic resonance spectra $\left({ }^{13} \mathrm{C} \mathrm{NMR}\right)$ were recorded at $100 \mathrm{MHz}$ or $125 \mathrm{MHz}$ and are reported $(\mathrm{ppm})$ relative to the center line of the triplet from chloroform- $d(77.23 \mathrm{ppm})$. Mass spectra were determined on a Finnigan Mat 95 high resolution gas chromatography/mass spectrometer with Finnigan Mat ICIS II operating system.

Ethyl Acetate Adducts 2a and 2b: Into a stirring solution of diisopropyl amine (3.66 $\mathrm{mL}, 26.1 \mathrm{mmol})$ in THF $(30 \mathrm{~mL})$ at $-78{ }^{\circ} \mathrm{C}$ was added $\mathrm{nBuLi}(10.9 \mathrm{~mL}$ of $2.4 \mathrm{M}$ solution, $26.1 \mathrm{mmol})$ and the resulting mixture was stirred at $-78^{\circ} \mathrm{C}$ for 30 minutes. This was then warmed to $0{ }^{\circ} \mathrm{C}$ over several minutes and stirred for another 30 minutes before being cooled once again to $-78^{\circ} \mathrm{C}$. Ethyl acetate $(2.44 \mathrm{~mL}, 25 \mathrm{mmol})$ was added dropwise, and the mixture was stirred for 1 hour. A solution of 2-[(2,2-dimethoxy)ethyl]cyclopentanone $1(4.0 \mathrm{~g}, 22.7 \mathrm{mmol})$ in THF $(10 \mathrm{~mL})$ was added dropwise to the above mixture and the 
reaction mixture was stirred at $-78^{\circ} \mathrm{C}$ until the reaction was complete by TLC analysis ( 4 h). The reaction mixture was quenched with saturated ammonium chloride $(25 \mathrm{~mL})$. After separation of the phases, the aqueous layer was extracted with $\mathrm{Et}_{2} \mathrm{O}(3 \times 15 \mathrm{~mL})$ and the combined organic extracts were washed with water $(50 \mathrm{~mL})$ and brine $(50 \mathrm{~mL})$, dried $\left(\mathrm{MgSO}_{4}\right)$, filtered and concentrated. The crude product $(5.64 \mathrm{~g}, 95.3 \%)$, a $1.7: 1$ mixture of inseparable cis and trans isomers $\mathbf{2 a}$ and $\mathbf{2 b}$ (ratio determined by ${ }^{1} \mathrm{H}$ NMR integration of methoxy singlets), was carried on without further purification.

2a: $\mathrm{R}_{f} 0.23$ (3:7 EtOAc/hexanes); IR ( $\mathrm{CH}_{2} \mathrm{Cl}_{2}$ cast) $3513,2953,2830,1731,1447,1371$, $1334,1190,1125,1057,964 \mathrm{~cm}^{-1}$; ${ }^{1} \mathrm{H}$ NMR $\left(500 \mathrm{MHz}, \mathrm{CDCl}_{3}\right) \square 4.37$ (dd, $J=7.8,3.5$ $\mathrm{Hz}, 1 \mathrm{H}), 4.14$ (q, $J=7.0 \mathrm{~Hz}, 2 \mathrm{H}) 3.28$ (s, 6H), 3.23 (br.s, $1 \mathrm{H}), 2.66$ (d, $\left.J_{\mathrm{AB}}=15.5 \mathrm{~Hz}, 1 \mathrm{H}\right)$, $2.33\left(\mathrm{~d}, J_{\mathrm{AB}}=15.5 \mathrm{~Hz}, 1 \mathrm{H}\right), 1.88-1.65(\mathrm{~m}, 5 \mathrm{H}), 1.60-1.50(\mathrm{~m}, 4 \mathrm{H}), 1.25(\mathrm{t}, J=7.0 \mathrm{~Hz}$, $3 \mathrm{H}) ;{ }^{13} \mathrm{C}$ NMR (125 MHz, $\left.\mathrm{CDCl}_{3}\right) \square 220.7,103.3,53.4,52.8,45.7,37.9,32.7,30.4,21.0$; LRMS (ESI) calcd for $\mathrm{C}_{13} \mathrm{H}_{24} \mathrm{O}_{5} \mathrm{Na}\left(\mathrm{M}^{+}\right)$283.1; found 283.1; Anal. Calcd for $\mathrm{C}_{13} \mathrm{H}_{24} \mathrm{O}_{5}$ : C, 59.98; H, 9.29. Found: C, 59.58; H, 10.01. (Data obtained from the inseparable mixture of diastereomerss)

2b: $\mathrm{R}_{f} 0.23$ (3:7 EtOAc/hexanes); IR ( $\mathrm{CH}_{2} \mathrm{Cl}_{2}$ cast) 3511, 2953, 2830, 1724, 1447, 1371, $1332,1199,1124,1058,966 \mathrm{~cm}^{-1} ;{ }^{1} \mathrm{H}$ NMR $\left(500 \mathrm{MHz}, \mathrm{CDCl}_{3}\right) \square 4.42(\mathrm{dd}, J=7.1,4.4$ $\mathrm{Hz}, 1 \mathrm{H}), 4.15(\mathrm{q}, J=7.2 \mathrm{~Hz}, 2 \mathrm{H}) 3.81(\mathrm{br} \mathrm{s}, 1 \mathrm{H}) 3.30$ and $3.26(2 \mathrm{~s}, 6 \mathrm{H}), 2.47\left(\mathrm{~d}, J_{\mathrm{AB}}=\right.$ $15.6 \mathrm{~Hz}, 1 \mathrm{H}), 2.38\left(\mathrm{~d}, J_{\mathrm{AB}}=15.6 \mathrm{~Hz}, 1 \mathrm{H}\right), 2.03-1.94(\mathrm{~m}, 2 \mathrm{H}), 1.82-1.76(\mathrm{ddd}, J=$ $13.9,7.1,4.2 \mathrm{~Hz}, 1 \mathrm{H}), 1.75-1.65(\mathrm{~m}, 3 \mathrm{H}), 1.61-1.51(\mathrm{~m}, 2 \mathrm{H}), 1.29-1.20(\mathrm{~m}, 2 \mathrm{H})$, $1.26(\mathrm{t}, J=7.2 \mathrm{~Hz}, 3 \mathrm{H}) ;{ }^{13} \mathrm{C} \mathrm{NMR}\left(125 \mathrm{MHz}, \mathrm{CDCl}_{3}\right) \square 173.7,103.8,80.7,61.0,53.5$, 52.2, 45.6, 39.7, 38.4, 33.2, 29.3, 20.8, 14.4; LRMS (ESI) calcd for $\mathrm{C}_{13} \mathrm{H}_{24} \mathrm{O}_{5} \mathrm{Na}\left(\mathrm{M}^{+}\right)$ 283.1; found 283.1; Anal. Calcd for $\mathrm{C}_{13} \mathrm{H}_{24} \mathrm{O}_{5}$ : C, 59.98; H, 9.29. Found: C, 59.85; H, 9.29. (Data obtained from a pure sample of $\mathbf{2} \mathbf{b}$ recovered after the formation of $\mathbf{3 a}$ from a mixture of $\mathbf{2 a}+\mathbf{2 b}$.)

Mixed Acetal 3a. To a solution of $\mathbf{2 a}$ and $\mathbf{2 b}(11.2 \mathrm{~g}, 43.3 \mathrm{mmol})$ in $\mathrm{CH}_{2} \mathrm{Cl}_{2}(1.0 \mathrm{~L})$ at $-15^{\circ} \mathrm{C}$ (acetone/ice bath) was added $\mathrm{BF}_{3} \bullet \mathrm{OEt}_{2}(5.50 \mathrm{~mL}, 43.3 \mathrm{mmol})$ and the reaction was stirred for 20 minutes, at which time TLC showed consumption of $\mathbf{2 a}$ and a mixture of $\mathbf{2 b}$ and 3a. The reaction was quenched with $\mathrm{Et}_{3} \mathrm{~N}(6 \mathrm{~mL})$ and water $(500 \mathrm{~mL})$ and the resulting phases were separated. The aqueous layer was extracted with $\mathrm{CH}_{2} \mathrm{Cl}_{2}$ and the combined organic extracts were washed with water and brine, then dried $\left(\mathrm{MgSO}_{4}\right)$, filtered and concentrated. The crude product was purified by column chromatography (silica gel; EtOAc/hexanes 3:7) to afford recovered 2b and 3a (3.85 g, 62 \%; 4.4:1 mixture of $\square$ and $\square$ anomers; ratio based on integration of OMe singlets) as a yellow oil: $\mathrm{R}_{f} 0.53$ (3:7 EtOAc/hexanes); IR $\left(\mathrm{CH}_{2} \mathrm{Cl}_{2}\right.$ cast) 2950, 2868, 2829, 1735, 1467, 1447, $1369,1342,1300,1208,1103,1049 \mathrm{~cm}^{-1}$; ${ }^{1} \mathrm{H}$ NMR $\left(500 \mathrm{MHz}, \mathrm{CDCl}_{3}\right) \square 4.97(\mathrm{~d}, J=5.2$ $\mathrm{Hz}, 0.8 \mathrm{H})$ overlapping with $4.96(\mathrm{~m}, 0.2 \mathrm{H}) 4.11(\mathrm{q}, J=7.1 \mathrm{~Hz}, 2 \mathrm{H}), 3.30(\mathrm{~s}, 2.4 \mathrm{H}), 3.28$ (s, $0.6 \mathrm{H}), 2.85\left(\mathrm{~d}, J_{\mathrm{AB}}=14.4 \mathrm{~Hz}, 0.8 \mathrm{H}\right), 2.67-2.61(\mathrm{~m}, 0.8 \mathrm{H}), 2.64\left(\mathrm{~d}, J_{\mathrm{AB}}=14.4 \mathrm{~Hz}\right.$, $0.2 \mathrm{H}), 2.63\left(\mathrm{~d}, J_{\mathrm{AB}}=14.4 \mathrm{~Hz}, 0.8 \mathrm{H}\right), 2.56(\mathrm{~m}, 0.2 \mathrm{H}), 2.52\left(\mathrm{~d}, J_{\mathrm{AB}}=14.4 \mathrm{~Hz}, 0.2 \mathrm{H}\right), 2.27$ (ddd, $J=13.3,9.5,5.7 \mathrm{~Hz}, 0.2 \mathrm{H}), 2.20(\mathrm{dd}, J=13.3 .9 .3 \mathrm{~Hz}, 0.8 \mathrm{H}) 1.96-1.87(\mathrm{~m}, 1 \mathrm{H})$, $1.73-1.56(\mathrm{~m}, 4.8 \mathrm{H}), 1.46-1.41(\mathrm{~m}, 1.2 \mathrm{H}), 1.24(\mathrm{t}, J=7.2 \mathrm{~Hz}, 2.4 \mathrm{H}), 1.23(\mathrm{t}, J=7.2 \mathrm{~Hz}$, $0.6 \mathrm{H}) ;{ }^{13} \mathrm{C}$ NMR $\left(125 \mathrm{MHz}, \mathrm{CDCl}_{3}\right)$ पMAJOR: 171.4, 106.7, 93.8, 60.4, 54.7, 46.2, 46.0, 41.4, 38.9, 33.6, 24.0, 14.5, MINOR: 171.2, 107.0, 94.9, 60.5, 54.9, 45.4, 44.9, 40.5, 
40.4, 34.2, 24.5, 14.5; HRMS (EI) calcd for $\mathrm{C}_{12} \mathrm{H}_{20} \mathrm{O}_{4}\left(\mathrm{M}^{+}\right)$228.1362; found 228.1359; Anal. Calcd for $\mathrm{C}_{12} \mathrm{H}_{20} \mathrm{O}_{4}$ : C, 63.14; H, 8.83. Found: C, 62.90; H, 9.43.

Mixed Thioacetal 3b. To a solution of 3a (50 mg, $0.22 \mathrm{mmol})$ and $p$-thiocresol $(27 \mathrm{mg}$, $0.22 \mathrm{mmol})$ in $\mathrm{CH}_{2} \mathrm{Cl}_{2}(22 \mathrm{~mL})$ at $-45^{\circ} \mathrm{C}$ was added $\mathrm{BF}_{3} \cdot \mathrm{OEt}_{2}(28 \square \mathrm{L}, 0.22 \mathrm{mmol})$ and the reaction was stirred until deemed complete by TLC. After 2 hours only a trace of $3 \mathbf{a}$ observed; the reaction was quenched with $\mathrm{Et}_{3} \mathrm{~N}(0.5 \mathrm{~mL})$ and water $(15 \mathrm{~mL})$ and the resulting bi-layer was separated. The aqueous phase was extracted with $\mathrm{CH}_{2} \mathrm{Cl}_{2}(15 \mathrm{~mL})$ and the combined organic extracts were washed with water $(30 \mathrm{~mL})$, brine $(30 \mathrm{~mL})$, dried with magnesium sulfate, filtered and concentrated. The crude product was first passed through a pad of silica gel in a fritted filter, eluting with $\mathrm{Et}_{2} \mathrm{O}$, and then purified by radial chromatography (silica gel, $2 \mathrm{~mm}$ plate, solvent ramp: $100 \mathrm{~mL}$ each of 3\%, 6\% then $9 \%$ EtOAc/hexanes until the product was recovered) to afford the product, a yellow oil, as a $1: 5: 1$ mixture of partially separable anomers $(3 \mathbf{b} \square$ and $\mathbf{3 b} \square)$ in $88 \%$ yield $(57 \mathrm{mg})$ based on recovered 3a $(4 \mathrm{mg})$.

Major Anomer 3b $\square: \mathrm{R}_{f} 0.63$ (3:7 EtOAc/hexanes); IR ( $\mathrm{CH}_{2} \mathrm{Cl}_{2}$ cast) 2954, 2867, 1731, 1493, 1446, 1369, 1340, 1300, 1195, 1094, 1054, $1034 \mathrm{~cm}^{-1} ;{ }^{1} \mathrm{H}$ NMR $(500 \mathrm{MHz}$, $\left.\mathrm{CDCl}_{3}\right) \square 7.35(\mathrm{~d}, J=8.2 \mathrm{~Hz}, 2 \mathrm{H}), 7.04(\mathrm{~d}, J=8.2 \mathrm{~Hz}, 2 \mathrm{H}), 5.51(\mathrm{dd}, J=6.9,5.4 \mathrm{~Hz}$, $1 \mathrm{H}), 4.09$ (q, $J=7.2 \mathrm{~Hz}, 2 \mathrm{H}), 2.94\left(\mathrm{~d}, J_{\mathrm{AB}}=14.6 \mathrm{~Hz}, 1 \mathrm{H}\right), 2.85\left(\mathrm{~d}, J_{\mathrm{AB}}=14.6 \mathrm{~Hz}, 1 \mathrm{H}\right)$, $2.74(\mathrm{ddd}, J=8.4,5.2,3.2 \mathrm{~Hz}, 1 \mathrm{H}), 2.29-2.24(\mathrm{~m}, 1 \mathrm{H}), 2.28(\mathrm{~s}, 3 \mathrm{H}), 2.08$ (ddd, $J=$ 13.6, 6.8, $5.2 \mathrm{~Hz}, 1 \mathrm{H}), 1.97-1.92(\mathrm{~m}, 1 \mathrm{H}), 1.84-1.76(\mathrm{~m}, 1 \mathrm{H}), 1.73-1.58(\mathrm{~m}, 3 \mathrm{H})$, $1.49-1.44(\mathrm{~m}, 1 \mathrm{H}), 1.22(\mathrm{t}, J=7.2 \mathrm{~Hz}, 3 \mathrm{H}) ;{ }^{13} \mathrm{C} \mathrm{NMR}\left(125 \mathrm{MHz}, \mathrm{CDCl}_{3}\right) \square 171.2$, 137.2, 132.2, 131.8, 129.8, 95.0, 88.3, 60.5, 46.9, 44.8, 41.0, 39.2, 33.3, 24.5, 21.3, 14.4; HRMS (EI) calcd for $\mathrm{C}_{18} \mathrm{H}_{24} \mathrm{O}_{3} \mathrm{~S}\left(\mathrm{M}^{+}\right)$320.1446; found 320.1419; Anal. Calcd for $\mathrm{C}_{18} \mathrm{H}_{24} \mathrm{O}_{3} \mathrm{~S}$ : C, 67.47; H, 7.55. Found: C, 67.24; H, 7.57.

Minor Anomer 3b $\square: \mathrm{R}_{f} 0.63$ (3:7 EtOAc/hexanes); IR $\left(\mathrm{CH}_{2} \mathrm{Cl}_{2}\right.$ cast) 3511, 2953, 2830, 1724, 1447, 1371, 1332, 1199, 1124, 1058, $966 \mathrm{~cm}^{-1} ;{ }^{1} \mathrm{H}$ NMR (500 MHz, $\left.\mathrm{CDCl}_{3}\right) \square 7.33$ $(\mathrm{d}, J=8.0 \mathrm{~Hz}, 2 \mathrm{H}), 7.04(\mathrm{~d}, J=8.0 \mathrm{~Hz}, 2 \mathrm{H}), 5.25(\mathrm{dd}, J=8.2,6.4 \mathrm{~Hz}, 1 \mathrm{H}), 4.09$ (q, $J=$ $7.2 \mathrm{~Hz}, 2 \mathrm{H}), 2.61(\mathrm{~s}, 2 \mathrm{H}), 2.66-2.53(\mathrm{~m}, 3 \mathrm{H}), 2.29(\mathrm{~s}, 3 \mathrm{H}), 2.04(\mathrm{~m}, 1 \mathrm{H}), 1.78-1.50$ $(\mathrm{m}, 6 \mathrm{H}), 1.22(\mathrm{t}, J=8.0 \mathrm{~Hz}, 3 \mathrm{H}) ;{ }^{13} \mathrm{C} \mathrm{NMR}\left(125 \mathrm{MHz}, \mathrm{CDCl}_{3}\right) \square 171.1,137.0,131.7$, 131.6, 129.7, 94.7, 86.2, 60.6, 46.8, 43.9, 41.0, 39.4, 33.8, 24.2, 21.3, 14.5; HRMS (EI) calcd for $\mathrm{C}_{18} \mathrm{H}_{24} \mathrm{O}_{3} \mathrm{~S}\left(\mathrm{M}^{+}\right) 320.1446$; found 320.1428 .

Diazoketones 4a. To a solution of mixed acetals 3a $(1.00 \mathrm{~g}, 4.38 \mathrm{mmol})$ in THF $(8 \mathrm{~mL})$ and methanol $(8 \mathrm{~mL})$ was added a $2.0 \mathrm{M}$ solution of $\mathrm{LiOH}(4.38 \mathrm{~mL})$. The reaction was stirred for 16 hours at room temperature, during which time the reaction mixture turned slightly yellow. The reaction was diluted with water $(20 \mathrm{~mL})$ and $\mathrm{Et}_{2} \mathrm{O}(20 \mathrm{~mL})$ and transferred to a separatory funnel. The layers were separated and the aqueous layer was washed with ether $(20 \mathrm{~mL})$. The aqueous layer was then acidified with $0.5 \mathrm{M} \mathrm{HCl}$ to $\mathrm{pH}$ $\sim 3$, resulting in a cloudy suspension. This was then diluted with ethyl acetate $(30 \mathrm{~mL})$ and the resulting layers separated. The aqueous layer was washed with 3 portions of ethyl acetate $(20 \mathrm{~mL})$ and the combined organic extracts were washed with water then brine, dried over magnesium sulfate, filtered and concentrated to give the acid (mixture of anomers) as a yellow oil (870 mg, 99\%): IR $\left(\mathrm{CH}_{2} \mathrm{Cl}_{2}\right.$ cast) $3600-2500,2949,1708$, 1468, 1440, 1409, 1339, 1300, $1218 \mathrm{~cm}^{-1} ;{ }^{1} \mathrm{H}$ NMR (500 MHz, $\left.\mathrm{CDCl}_{3}\right) \square 10.8$ (br s, $\left.1 \mathrm{H}\right)$, $4.99(\mathrm{~d}, J=5.2 \mathrm{~Hz}, 1.0 \mathrm{H}), 3.30(\mathrm{~s}, 2.4 \mathrm{H}), 3.28(\mathrm{~s}, 0.6 \mathrm{H}), 2.90\left(\mathrm{~d}, J_{\mathrm{AB}}=14.9 \mathrm{~Hz}, 0.8 \mathrm{H}\right)$, 
$2.64\left(\mathrm{~d}, J_{\mathrm{AB}}=14.6 \mathrm{~Hz}, 0.2 \mathrm{H}\right), 2.63\left(\mathrm{~d}, J_{\mathrm{AB}}=14.9 \mathrm{~Hz}, 0.8 \mathrm{H}\right), 2.56\left(\mathrm{~d}, J_{\mathrm{AB}}=14.6 \mathrm{~Hz}\right.$, $0.2 \mathrm{H}$ ), 2.49 (ddd, $J=4.6,1.2 \mathrm{~Hz}, 0.2 \mathrm{H}), 2.27$ (ddd, $J=13.6,9.8,5.7 \mathrm{~Hz}, 0.2 \mathrm{H}), 2.20$ (dd, $J=13.3,9.2 \mathrm{~Hz}, 0.8 \mathrm{H}), 1.98-1.80(\mathrm{~m}, 1.6 \mathrm{H}), 1.75-1.55(\mathrm{~m}, 5.2 \mathrm{H}), 1.43(\mathrm{~m}, 1.0 \mathrm{H}) ;{ }^{13} \mathrm{C}$ NMR (125 MHz, $\left.\mathrm{CDCl}_{3}\right) \square$ Major Anomer: 176.0, 107.1, 93.5, 55.0, 46.5, 46.0, 41.1, 38.9, 33.7, 24.0; Minor Anomer: 176.0, 107.2, 94.7, 55.1, 44.8, 40.2, 40.1, 34.0, 24.6; HRMS (EI) calcd for $\mathrm{C}_{10} \mathrm{H}_{16} \mathrm{O}_{4}\left(\mathrm{M}^{+}\right)$200.1049; found 200.1051.

To a solution of the anomeric acids $(800 \mathrm{mg}, 4.0 \mathrm{mmol})$ in $\mathrm{Et}_{2} \mathrm{O}(50 \mathrm{~mL})$ at $0^{\circ} \mathrm{C}$ was added $\mathrm{Et}_{3} \mathrm{~N}$ (585 $\square \mathrm{L}, 4.4 \mathrm{mmol}$ ) followed by isobutyl chloroformate (574 $\square \mathrm{L}, 4.4 \mathrm{mmol}$ ) and the reaction was stirred for 2.5 hours, resulting in the formation of an off-white precipitate. The reaction mixture was filtered through a fritted filter (D), rinsed with $\mathrm{Et}_{2} \mathrm{O}(50 \mathrm{~mL})$ and concentrated to give the mixed anhydride $(1.09 \mathrm{~g}, 91 \%)$ as a yellow oil: $\mathrm{R}_{f} 0.62$ (3:7 EtOAc/hexanes); IR ( $\mathrm{CH}_{2} \mathrm{Cl}_{2}$ cast) 2958, 2784, 2831, 1804, 1760, 1470, 1452, 1396, $1370 \mathrm{~cm}^{-1} ;{ }^{1} \mathrm{H}$ NMR (500 MHz, $\left.\mathrm{CDCl}_{3}\right)$ Major Anomer $\square 5.00(\mathrm{~d}, J=5.3 \mathrm{~Hz}$, $1 \mathrm{H}), 4.03(\mathrm{~d}, J=7.1 \mathrm{~Hz}, 2 \mathrm{H}), 3.31(\mathrm{~s}, 3 \mathrm{H}), 3.03\left(\mathrm{~d}, J_{\mathrm{AB}}=15.3 \mathrm{~Hz}, 1 \mathrm{H}\right), 2.80\left(\mathrm{~d}, J_{\mathrm{AB}}=\right.$ $15.3 \mathrm{~Hz}, 1 \mathrm{H}), 2.66(\mathrm{~m}, 1 \mathrm{H}), 2.23(\mathrm{dd}, J=13.3,9.2 \mathrm{~Hz}, 1 \mathrm{H}), 2.01$ (sept, $J=6.8 \mathrm{~Hz}, 1 \mathrm{H})$ $2.00(\mathrm{~m}, 1 \mathrm{H}), 1.76(\mathrm{~m}, 1 \mathrm{H}), 1.68(\mathrm{ddd}, J=12.0,7.5,5.3 \mathrm{~Hz}, 1 \mathrm{H}), 1.63(\mathrm{~m}, 3 \mathrm{H}), 1.46(\mathrm{~m}$, $1 \mathrm{H}), 0.95(\mathrm{~d}, J=6.8 \mathrm{~Hz}, 6 \mathrm{H}) ;{ }^{13} \mathrm{C} \mathrm{NMR}\left(125 \mathrm{MHz}, \mathrm{CDCl}_{3}\right) \square$ Major Anomer: 165.8 , 149.4, 106.9, 93.1, 75.7, 54.8, 46.4, 45.7, 41.3, 38.8, 33.5, 27.8, 24.0, 19.0; Minor Anomer: 165.6, 149.4, 106.7, 94.4, 75.5, 54.8, 45.6, 44.6, 41.4, 38.9, 34.3, 33.6, 24.5, 19.0; HRMS (EI) calcd for $\mathrm{C}_{15} \mathrm{H}_{23} \mathrm{O}_{6}\left(\mathrm{M}-\mathrm{H}^{+}\right)$299.1495; found 299.1499.

A solution of anomeric mixed anhydrides (624 mg, $2.2 \mathrm{mmol})$ in $\mathrm{Et}_{2} \mathrm{O}(10 \mathrm{~mL})$ was added via cannula to a solution of freshly prepared diazomethane $(20 \mathrm{mmol})$ in $\mathrm{Et}_{2} \mathrm{O}(60$ $\mathrm{mL}$ ) at $-15^{\circ} \mathrm{C}$, and the resulting mixture stirred for 16 hours as the cooling bath expired. A gentle stream of $\mathrm{N}_{2}$ was applied to the system to allow for slow evaporation of both excess diazomethane and solvent, and the resulting yellow oil was diluted in ether (20 $\mathrm{mL}$ ), passed through a short pad of silica gel in a fritted filter, eluting with copious ether. This was then concentrated and the resulting oil purified by radial chromatography (silica gel, $4 \mathrm{~mm}$ plate, solvent ramp: $100 \mathrm{~mL}$ each of 3\%, 6\%, 9\% then $12 \%$ EtOAc/hexanes until the products were recovered) to yield $\mathbf{4 a}$, an inseparable 4.7:1 mixture of anomers, (228 mg, $49 \%$ ) as a bright yellow oil, as well as a trace of starting material (not quantified): $\mathrm{R}_{f} 0.23$ (3:7 EtOAc/hexanes); IR $\left(\mathrm{CH}_{2} \mathrm{Cl}_{2}\right.$ cast) $3087,2949,2868,2829$, 2102, 1817, 1735, 1637, 1440, $1361 \mathrm{~cm}^{-1} ;{ }^{1} \mathrm{H} \mathrm{NMR}\left(500 \mathrm{MHz},-40{ }^{\circ} \mathrm{C} \mathrm{CDCl} \mathrm{CD}_{3}\right) \square 5.67(\mathrm{~s}$, $0.8 \mathrm{H}), 5.52(\mathrm{~s}, 0.2 \mathrm{H}), 5.01(\mathrm{~m}, J=5.1 \mathrm{~Hz}, 1 \mathrm{H}), 3.36(\mathrm{~s}, 2.4 \mathrm{H}), 3.30(\mathrm{~s}, 0.6 \mathrm{H}), 2.94\left(\mathrm{~d}, J_{\mathrm{AB}}\right.$ $=14.3 \mathrm{~Hz}, 0.8 \mathrm{H}), 2.69\left(\mathrm{~d}, J_{\mathrm{AB}}=13.5 \mathrm{~Hz}, 0.2 \mathrm{H}\right), 2.60(\mathrm{~m}, 1 \mathrm{H}), 2.55\left(\mathrm{~d}, J_{\mathrm{AB}}=14.3 \mathrm{~Hz}\right.$, $0.8 \mathrm{H}), 2.47\left(\mathrm{~d}, J_{\mathrm{AB}}=13.5 \mathrm{~Hz}, 0.2 \mathrm{H}\right), 2.20(\mathrm{dd}, J=13.5,9.5 \mathrm{~Hz}, 1 \mathrm{H}), 1.90(\mathrm{~m}, 1 \mathrm{H}), 1.70-$ 1.45 (m, 6H); ${ }^{13} \mathrm{C}$ NMR (125 MHz, $\mathrm{CDCl}_{3},-60{ }^{\circ} \mathrm{C}$ ) Major Anomer: $\square$ 194.1, 106.4, 93.4, 56.0, 54.9, 52.8, 46.4, 40.3, 38.1, 32.5, 23.4; Minor Anomer: $\square$ 194.1, 106.5, 95.4, 56.8, $54.7,50.8,44.2,40.3,39.9,33.6,24.2$; HRMS (ESI) calcd for $\mathrm{C}_{11} \mathrm{H}_{16} \mathrm{~N}_{2} \mathrm{O}_{3} \mathrm{Na}\left(\mathrm{M}^{+}\right)$ 247.1059 ; found 247.1060 .

Diazoketones 4b. Preparation and characterization of individual acid anomers is given below. For convenience, they were usually prepared as a mixture of anomers and converted into the separable diazoketones, $4 \mathrm{~b} \square$ and $4 \mathrm{~b} \square$. 
To a solution of mixed acetal $3 \mathrm{~b} \square(423 \mathrm{mg}, 1.3 \mathrm{mmol})$ in THF $(5 \mathrm{~mL})$ and methanol (5 $\mathrm{mL}$ ) was added a $2.0 \mathrm{M}$ solution of $\mathrm{LiOH}(1.3 \mathrm{~mL})$. The reaction was stirred for 16 hours at room temperature, during which time the reaction mixture turned slightly yellow. The reaction was diluted with water $(20 \mathrm{~mL})$ and $\mathrm{Et}_{2} \mathrm{O}(20 \mathrm{~mL})$ and transferred to a separatory funnel. The layers were separated and the aqueous layer was washed with ether $(15 \mathrm{~mL})$. The aqueous layer was then acidified with $0.5 \mathrm{M} \mathrm{HCl}$ to $\mathrm{pH} \sim 3$, resulting in a cloudy suspension. This was then diluted with ethyl acetate $(40 \mathrm{~mL})$ and the resulting layers separated. The aqueous layer was washed with 3 portions of ethyl acetate $(20 \mathrm{~mL})$ and the combined organic extracts were washed with water $(50 \mathrm{~mL})$ then brine $(50 \mathrm{~mL})$, dried over magnesium sulfate, filtered and concentrated to yield a yellow oil (333 $\mathrm{mg}, 86 \%$ ): IR $\left(\mathrm{CH}_{2} \mathrm{Cl}_{2}\right.$ cast $) 3500-2400,2953,2867,1708,1597,1493,1443,1408,1300,1231$. $1133,1093 \mathrm{~cm}^{-1} ;{ }^{1} \mathrm{H}$ NMR $\left(500 \mathrm{MHz}, \mathrm{CDCl}_{3}\right) \square 7.34(\mathrm{~d}, J=8.2 \mathrm{~Hz}, 2 \mathrm{H}), 7.07(\mathrm{~d}, J=8.2$ $\mathrm{Hz}, 2 \mathrm{H}), 5.56(\mathrm{dd}, J=6.0,6.0 \mathrm{~Hz}, 1 \mathrm{H}), 3.03\left(\mathrm{~d}, J_{\mathrm{AB}}=14.8 \mathrm{~Hz}, 1 \mathrm{H}\right), 2.74\left(\mathrm{~d}, J_{\mathrm{AB}}=14.8\right.$ $\mathrm{Hz}, 1 \mathrm{H}), 2.63(\mathrm{~m}, 1 \mathrm{H}), 2.32-2.25(\mathrm{~m}, 1 \mathrm{H}), 2.29$ (s, 3H), 2.14 (ddd, $J=13.4,6.8,5.0 \mathrm{~Hz}$, $1 \mathrm{H}), 1.98-1.92(\mathrm{~m}, 1 \mathrm{H}), 1.88-1.79(\mathrm{~m}, 1 \mathrm{H}), 1.70-1.58(\mathrm{~m}, 3 \mathrm{H}), 1.52-1.46(\mathrm{~m}, 1 \mathrm{H})$, (COOH peak not measured); ${ }^{13} \mathrm{C}$ NMR $\left(125 \mathrm{MHz}, \mathrm{CDCl}_{3}\right) \square 186.8,137.9,132.5,130.8$, $130.0,94.7,88.7,47.5,44.9,40.5,39.0,33.0,24.5,21.3$; HRMS (EI) calcd for $\mathrm{C}_{16} \mathrm{H}_{20} \mathrm{SO}_{3}\left(\mathrm{M}^{+}\right)$292.1133; found 292.1135.

Following the same procedure as above, $\mathbf{3 b} \square(142 \mathrm{mg}, 0.44 \mathrm{mmol})$ was converted into the corresponding acid (100 mg, 78\%) as a white solid: m.p. $115-116^{\circ} \mathrm{C}$; $\mathrm{IR}\left(\mathrm{CH}_{2} \mathrm{Cl}_{2}\right.$ cast) $3500-2500,2949,2865,1707,1493,1439,1411,1299,1232.1146,1093 \mathrm{~cm}^{-1} ;{ }^{1} \mathrm{H}$ NMR (500 MHz, $\left.\mathrm{CDCl}_{3}\right) \square 9.4($ br s, $1 \mathrm{H}), 7.34(\mathrm{~d}, J=8.4 \mathrm{~Hz}, 2 \mathrm{H}), 7.06(\mathrm{~d}, J=8.4 \mathrm{~Hz}$, $2 \mathrm{H}), 5.28(\mathrm{dd}, J=8.2,5.6 \mathrm{~Hz}, 1 \mathrm{H}), 2.69\left(\mathrm{~d}, J_{\mathrm{AB}}=14.8 \mathrm{~Hz}, 1 \mathrm{H}\right), 2.57\left(\mathrm{~d}, J_{\mathrm{AB}}=14.8 \mathrm{~Hz}\right.$, $1 \mathrm{H}), 2.60-2.51(\mathrm{~m}, 2 \mathrm{H}), 2.29(\mathrm{~s}, 3 \mathrm{H}), 2.10(\mathrm{~m}, 1 \mathrm{H}), 1.78-1.54(\mathrm{~m}, 6 \mathrm{H}) ;{ }^{13} \mathrm{C}$ NMR $(125$ $\left.\mathrm{MHz}, \mathrm{CDCl}_{3}\right) \square 186.8,137.7,132.4,130.7,129.9,94.4,86.5,47.6,43.6,40.6,39.0,33.6$, 24.3, 21.3; HRMS (EI) calcd for $\mathrm{C}_{16} \mathrm{H}_{20} \mathrm{SO}_{3}\left(\mathrm{M}^{+}\right)$292.1133; found 292.1141; Anal. Calcd for $\mathrm{C}_{16} \mathrm{H}_{20} \mathrm{O}_{3} \mathrm{~S}: \mathrm{C}, 65.72 ; \mathrm{H}, 6.89$. Found: $\mathrm{C}, 65.21 ; \mathrm{H}, 7.07$.

To a solution of both anomers of the acids derived from $3 \mathbf{b} \square$ and $\mathbf{3 b} \square(1.0 \mathrm{~g}, 3.42 \mathrm{mmol})$ in $\mathrm{CH}_{2} \mathrm{Cl}_{2}(140 \mathrm{~mL})$ at $-15^{\circ} \mathrm{C}$ was added 2,6 -lutidine $(478 \square \mathrm{L}, 4.1 \mathrm{mmol})$ followed by oxalyl chloride ( $36 \square \mathrm{L}, 4.1 \mathrm{mmol}$ ) and DMF (2 drops, $\sim 10 \square \mathrm{L}$ ), resulting in the evolution of copious amounts of gas. The reaction was stirred for 4 hours, and solvent was removed by rotary evaporation to give a yellow oil entrained in a white precipitate. This material was redissolved in ether $(50 \mathrm{~mL})$ and the suspension filtered through a fritted filter (D), washing several times with ether $(50 \mathrm{~mL})$. The ethereal solution of the acid chloride was condensed to a lesser volume $(\sim 50 \mathrm{~mL})$ and added via cannula to a solution of freshly prepared diazomethane $(60 \mathrm{mmol})$ in $\mathrm{Et}_{2} \mathrm{O}(200 \mathrm{~mL})$ at $-15^{\circ} \mathrm{C}$, and the resulting mixture stirred for 16 hours as the cooling bath expired. A gentle stream of $\mathrm{N}_{2}$ was applied to the system to allow for slow evaporation of both excess diazomethane and solvent, and the resulting yellow oil was diluted in ether $(40 \mathrm{~mL})$, passed through a short pad of silica gel in a fritted filter, eluting with copious ether. The filtrate was concentrated and the resulting oil purified by radial chromatography (silica gel, $4 \mathrm{~mm}$ plate, solvent ramp: $100 \mathrm{~mL}$ each of $3 \%, 6 \%, 9 \%$ then $12 \%$ EtOAc/hexanes until the products were recovered) to yield $4 \mathrm{~b} \square(410 \mathrm{mg})$ and $4 \mathrm{~b} \square(240 \mathrm{mg})$, as yellow oils in a combined overall yield of $60 \%$. 
Major Anomer 4b $\square$ : $\mathrm{R}_{f} 0.32$ (3:7 EtOAc/hexanes); IR $\left(\mathrm{CH}_{2} \mathrm{Cl}_{2}\right.$ cast) 3105, 2953, 2866, $2100,1636,1492,1450,1358,1241,1162,1093,1061,1017 \mathrm{~cm}^{-1} ;{ }^{1} \mathrm{H}$ NMR $(500 \mathrm{MHz},-$ $\left.60{ }^{\circ} \mathrm{C}, \mathrm{CDCl}_{3}\right) \square 7.36(\mathrm{~d}, J=8.0 \mathrm{~Hz}, 2 \mathrm{H}), 7.14(\mathrm{~d}, J=8.0 \mathrm{~Hz}, 2 \mathrm{H}), 5.81(\mathrm{dd}, J=6.2,2.7$ $\mathrm{Hz}, 1 \mathrm{H}), 5.12(\mathrm{~s}, 1 \mathrm{H}), 3.23\left(\mathrm{~d}, J_{\mathrm{AB}}=13.2 \mathrm{~Hz}, 1 \mathrm{H}\right), 2.66(\operatorname{app} . \mathrm{q}, J=7.3 \mathrm{~Hz}, 1 \mathrm{H}), 2.49(\mathrm{~d}$, $\left.J_{\mathrm{AB}}=13.2 \mathrm{~Hz}, 1 \mathrm{H}\right), 2.37-2.30(\mathrm{~m}, 1 \mathrm{H}), 2.32(\mathrm{~s}, 3 \mathrm{H}), 2.17-2.11(\mathrm{~m}, 1 \mathrm{H}), 1.84-1.52$ $(\mathrm{m}, 6 \mathrm{H}) ;{ }^{13} \mathrm{C}$ NMR $\left(125 \mathrm{MHz},-60^{\circ} \mathrm{C}, \mathrm{CDCl}_{3}\right) \square 194.3,136.5,131.3,129.8,129.8,95.0$, 87.0, 56.2, 52.1, 47.4, 29.8, 98.0, 32.2, 23.5, 21.1; HRMS (ESI) calcd for $\mathrm{C}_{17} \mathrm{H}_{20} \mathrm{~N}_{2} \mathrm{O}_{2} \mathrm{SNa}\left(\mathrm{M}^{+}\right)$339.1143; found 339.1147; Anal. Calcd for $\mathrm{C}_{17} \mathrm{H}_{20} \mathrm{~N}_{2} \mathrm{O}_{2} \mathrm{~S}$ : C, 64.53; H, 6.37; N, 8.85. Found: C, 64.92; H, 6.13; N, 8.66.

Minor Anomer 4b $\square: \mathrm{R}_{f} 0.26$ (3:7 EtOAc/hexanes); IR ( $\mathrm{CH}_{2} \mathrm{Cl}_{2}$ cast) 3081, 2952, 2865 , $2100,1636,1492,1449,1358,1161,1093,1051,1016 \mathrm{~cm}^{-1} ;{ }^{1} \mathrm{H}$ NMR $\left(500 \mathrm{MHz},-60{ }^{\circ} \mathrm{C}\right.$, $\left.\mathrm{CDCl}_{3}\right) \square 7.39(\mathrm{~d}, J=8.0 \mathrm{~Hz}, 2 \mathrm{H}), 7.13(\mathrm{~d}, J=8.0 \mathrm{~Hz}, 2 \mathrm{H}), 5.53(\mathrm{~s}, 1 \mathrm{H}), 5.14(\mathrm{dd}, J=$ 9.7, $5.4 \mathrm{~Hz}, 1 \mathrm{H}), 2.66\left(\mathrm{~d}, J_{\mathrm{AB}}=13.6 \mathrm{~Hz}, 1 \mathrm{H}\right), 2.60(\operatorname{app} . \mathrm{q}, J=7.6 \mathrm{~Hz}, 1 \mathrm{H}), 2.53\left(\mathrm{~d}, J_{\mathrm{AB}}=\right.$ $13.6 \mathrm{~Hz}, 1 \mathrm{H}), 2.48$ (ddd, $J=13.0,9.0,5.5 \mathrm{~Hz}, 1 \mathrm{H}), 2.34$ (s, 3H), $2.04-1.98$ (br m, $1 \mathrm{H})$, $1.66-1.46(\mathrm{~m}, 6 \mathrm{H}) ;{ }^{13} \mathrm{C}$ NMR $\left(125 \mathrm{MHz},-60^{\circ} \mathrm{C} \mathrm{CDCl}_{3}\right) \square 193.8,137.5,131.8,129.7$, 129.5, 94.0, 84.9, 56.7, 50.0, 46.8, 39.6, 38.5, 32.7, 23.4, 21.2; HRMS (ESI) calcd for $\mathrm{C}_{17} \mathrm{H}_{20} \mathrm{~N}_{2} \mathrm{O}_{2} \mathrm{SNa}\left(\mathrm{M}^{+}\right)$339.1143; found 339.1143; Anal. Calcd for $\mathrm{C}_{17} \mathrm{H}_{20} \mathrm{~N}_{2} \mathrm{O}_{2} \mathrm{~S}$ : C, 64.53; H, 6.37; N, 8.85. Found: C, 64.40; H, 6.58; N, 8.52.

Carbene Transfer Reaction of 4a; Preparation of 7a $\square$ and 7a $\square$. To a refluxing solution of $\mathrm{Cu}(\mathrm{hfacac})_{2}(37 \mathrm{mg}, 0.08 \mathrm{mmol}, 10 \mathrm{~mol} \%)$ in $\mathrm{CH}_{2} \mathrm{Cl}_{2}(55 \mathrm{~mL})$ was added a solution of $4 \mathbf{a}$ (168 mg, $0.75 \mathrm{mmol}, 4.4: 1$ mixture of anomers) in $\mathrm{CH}_{2} \mathrm{Cl}_{2}(25 \mathrm{~mL})$, and the resulting mixture monitored by TLC. Upon consumption of diazoketone $4 \mathbf{a}$ (45 min) the reaction mixture was cooled to room temperature and quenched with $0.5 \mathrm{M} \mathrm{K}_{2} \mathrm{CO}_{3}$ $(25 \mathrm{~mL})$. The layers were separated and the aqueous phase extracted with $\mathrm{CH}_{2} \mathrm{Cl}_{2}(2 \times 15$ $\mathrm{mL})$. The organic extracts were combined and washed with water $(20 \mathrm{~mL})$, pre-dried with brine $(20 \mathrm{~mL})$, dried with magnesium sulfate, filtered and concentrated. The resulting pale oil was purified by gradient column chromatograhy (silica gel; $5 \%, 10 \%$, $15 \%$ then $20 \% \mathrm{EtOAc} /$ hexanes until the products were recovered) to yield $7 \mathrm{a} \square(68 \mathrm{mg})$ and $7 \mathbf{a} \square(25 \mathrm{mg}$ ) as pale yellow oils, in an overall yield of $67 \%$. (This reaction was also carried out on small scale with pure samples of $4 \mathrm{a} \square$ and $4 \mathrm{a} \square$. $4 \mathrm{a} \square$ also furnished trace amounts of product $\mathbf{A}$ [see footnote 9 in the text].)

Major Diastereomer 7a $\square$ : $\mathrm{R}_{f} 0.40$ (3:7 EtOAc/hexanes); IR $\left(\mathrm{CH}_{2} \mathrm{Cl}_{2}\right.$ cast) 2938, 2871, $2825,1760,1456,1403,1370,1347,1312 \mathrm{~cm}^{-1} ;{ }^{1} \mathrm{H}$ NMR $\left(500 \mathrm{MHz}, \mathrm{CDCl}_{3}\right) \square 4.04(\mathrm{~d}, J$ $=4.9 \mathrm{~Hz}, 1 \mathrm{H}), 3.61(\mathrm{ddd}, J=10.6,5.3,5.3 \mathrm{~Hz}, 1 \mathrm{H}), 3.34(\mathrm{~s}, 3 \mathrm{H}), 2.54\left(\mathrm{~d}, J_{\mathrm{AB}}=17.6 \mathrm{~Hz}\right.$, $1 \mathrm{H}), 2.19\left(\mathrm{~d}, J_{\mathrm{AB}}=17.6 \mathrm{~Hz}, 1 \mathrm{H}\right), 2.06(\mathrm{dd}, J=13.9,5.7 \mathrm{~Hz}, 1 \mathrm{H}), 1.92-1.76(\mathrm{~m}, 5 \mathrm{H})$, $1.76-1.69(\mathrm{~m}, 1 \mathrm{H}), 1.68-1.62(\mathrm{~m}, 1 \mathrm{H}), 1.56(\mathrm{ddd}, J=13.9,9.1 .6 .0 \mathrm{~Hz}, 1 \mathrm{H}) ;{ }^{13} \mathrm{C} \mathrm{NMR}$ $\left(125 \mathrm{MHz}, \mathrm{CDCl}_{3}\right) \square$ 214.4, 88.7, 78.3, 74.7, 56.8, 44.6, 44.4, 34.1, 28.9, 27.5, 21.3; HRMS (ESI) calcd for $\mathrm{C}_{11} \mathrm{H}_{16} \mathrm{O}_{3}\left(\mathrm{M}^{+}\right)$196.1099; found 196.1097.

Minor Anomer 7a $\square$ : $\mathrm{R}_{f} 0.46$ (3:7 EtOAc/hexanes); IR $\left(\mathrm{CH}_{2} \mathrm{Cl}_{2}\right.$ cast) 2938, 2872, 2824, 1761, 1436, 1405, 1368, 1347, $1312 \mathrm{~cm}^{-1} ;{ }^{1} \mathrm{H}$ NMR (500 MHz, $\left.\mathrm{CDCl}_{3}\right) \square 4.19(\mathrm{~s}, 1 \mathrm{H})$, $3.28(\mathrm{~s}, 3 \mathrm{H}), 3.23(\mathrm{~m}, 1 \mathrm{H}), 2.60\left(\mathrm{~d}, J_{\mathrm{AB}}=17.3 \mathrm{~Hz}, 1 \mathrm{H}\right), 2.22\left(\mathrm{~d}, J_{\mathrm{AB}}=17.3 \mathrm{~Hz}, 1 \mathrm{H}\right), 2.03$ - $1.74(\mathrm{~m}, 7 \mathrm{H}), 1.66-1.54(\mathrm{~m}, 2 \mathrm{H}) ;{ }^{13} \mathrm{C}$ NMR (125 MHz, $\left.\mathrm{CDCl}_{3}\right) \square 215.8,88.7,80.3$, 75.3, 56.4, 45.3, 41.9, 34.9, 30.9, 26.4, 21.8; HRMS (ESI) calcd for $\mathrm{C}_{11} \mathrm{H}_{16} \mathrm{O}_{3}\left(\mathrm{M}^{+}\right)$ 196.1099; found 196.1092. 
Side Product A (partial data): ${ }^{1} \mathrm{H}$ NMR $\left(500 \mathrm{MHz}, \mathrm{CDCl}_{3}\right) \square 6.03(\mathrm{~d}, J=2.3 \mathrm{~Hz}, 1 \mathrm{H})$, $5.56(\mathrm{~d}, J=4.5 \mathrm{~Hz}, 1 \mathrm{H}), 3.49$ (s, 3H), $2.54(\mathrm{dd}, J=14.1,2.3 \mathrm{~Hz}, 1 \mathrm{H}), 2.42$ (d, $J=14.1$ $\mathrm{Hz}, 1 \mathrm{H}), 2.33(\mathrm{dd}, J=13.2,8.9 \mathrm{~Hz}, 1 \mathrm{H}), 2.24(\mathrm{dddd}, J=9.8,8.7,6.4,4.0 \mathrm{~Hz}, 1 \mathrm{H}), 2.01-$ $1.83(\mathrm{~m}, 3 \mathrm{H}), 1.74(\mathrm{app} \mathrm{dt}, J=13.2,4.3 \mathrm{~Hz}, 1 \mathrm{H}), 1.70-1.56(\mathrm{~m}, 2 \mathrm{H}), 1.35-1.27(\mathrm{~m}, 1 \mathrm{H})$; ${ }^{13} \mathrm{C}$ NMR $\left(125 \mathrm{MHz}, \mathrm{CDCl}_{3}\right) \square 136.2,134.0,102.9,93.2,60.1,44.1,40.9,36.0,34.2$, $33.1,25.7$.

Carbene Transfer Reaction of $4 \mathrm{~b} \square$; Preparation of $7 \mathrm{~b} \square, 7 \mathrm{~b} \square$ and 8. To a refluxing solution of $\mathrm{Cu}(\mathrm{hfacac})_{2}(16 \mathrm{mg}, 0.03 \mathrm{mmol}, 10 \mathrm{~mol} \%)$ in $\mathrm{CH}_{2} \mathrm{Cl}_{2}(33 \mathrm{~mL})$ was added a solution of $4 \mathrm{~b} \square(105 \mathrm{mg}, 0.33 \mathrm{mmol})$ in $\mathrm{CH}_{2} \mathrm{Cl}_{2}(8 \mathrm{~mL})$, and the resulting mixture monitored by TLC. Upon consumption of diazoketone $4 \mathrm{~b} \square$ (30 min) the reaction mixture was cooled to room temperature and quenched with $0.5 \mathrm{M} \mathrm{K}_{2} \mathrm{CO}_{3}(25 \mathrm{~mL})$. The layers were separated and the aqueous phase extracted with $\mathrm{CH}_{2} \mathrm{Cl}_{2}(2 \times 15 \mathrm{~mL})$. The organic extracts were combined and washed with water $(25 \mathrm{~mL})$, pre-dried with brine $(25$ $\mathrm{mL}$ ), dried with magnesium sulfate, filtered and concentrated. The resulting pale yellow oil was diluted in ether $(20 \mathrm{~mL})$, passed through a short pad of silica gel in a fritted filter, rinsing with excess ether. This was then concentrated and the resulting oil purified by radial chromatography (silica gel, $2 \mathrm{~mm}$ plate, solvent ramp: $100 \mathrm{~mL}$ each of $2 \%, 5 \%$, $7 \%$ then $10 \%$ EtOAc/hexanes until the products were recovered) to yield a mixture of $7 \mathrm{~b} \square$ and $7 \mathrm{~b} \square(59 \mathrm{mg}, 68 \%$, ca. 2:1 ratio) as pale yellow oils, as well as 8 (11 $\mathrm{mg}, 13 \%)$ as a white solid, in an overall yield of $80 \%$. Minor diastereomer $7 \mathbf{b} \square$ could be obtained as a white solid, m.p.: $64-65^{\circ} \mathrm{C}$.

Diastereomers $7 \mathrm{~b} \square$ and $7 \mathrm{~b} \square: \mathrm{R}_{f} 0.41$ (3:7 EtOAc/hexanes); IR ( $\mathrm{CH}_{2} \mathrm{Cl}_{2}$ cast) 3019, 2955, 2869, 1785, 1711, 1492, 1447, 1401, $1343 \mathrm{~cm}^{-1} ;{ }^{1} \mathrm{H}$ NMR (500 MHz, $\left.\mathrm{CDCl}_{3}\right) \square$ $7.35(\mathrm{~d}, J=8.1 \mathrm{~Hz}, 2 \mathrm{H}), 7.28(\mathrm{~d}, J=8.1 \mathrm{~Hz}, 1 \mathrm{H}), 7.07(\mathrm{~d}, J=8.1 \mathrm{~Hz}, 1 \mathrm{H}), 7.06(\mathrm{~d}, J=$ $8.1 \mathrm{~Hz}, 2 \mathrm{H}), 4.01(\mathrm{~s}, 0.5 \mathrm{H}), 3.86(\mathrm{~d}, J=3.9 \mathrm{~Hz}, 1 \mathrm{H}), 3.50(\mathrm{ddd}, J=13.2,5.4,4.2 \mathrm{~Hz}$, $1 \mathrm{H}), 3.27$ (ddd, $J=6.9,13.5,1.7 \mathrm{~Hz}, 0.5 \mathrm{H}), 2.60\left(\mathrm{~d}, J_{\mathrm{AB}}=17.3 \mathrm{~Hz}, 0.5 \mathrm{H}\right), 2.57\left(\mathrm{~d}, J_{\mathrm{AB}}=\right.$ $17.6 \mathrm{~Hz}, 1 \mathrm{H}), 2.29(\mathrm{~s}, 4.5 \mathrm{H}), 2.24\left(\mathrm{~d}, J_{\mathrm{AB}}=17.3 \mathrm{~Hz}, 0.5 \mathrm{H}\right), 2.23\left(\mathrm{~d}, J_{\mathrm{AB}}=17.6 \mathrm{~Hz}, 1 \mathrm{H}\right)$, $2.16-2.06(\mathrm{~m}, 2 \mathrm{H}), 1.96-1.77(\mathrm{~m}, 7.5 \mathrm{H}), 1.75-1.62(\mathrm{~m}, 4 \mathrm{H}) ;{ }^{13} \mathrm{C}$ NMR $(125 \mathrm{MHz}$, $\left.\mathrm{CDCl}_{3}\right) \square$ Major Diast: 213.5, 138.0, 133.3, 130.1, 129.9, 88.5, 79.8, 44.8, 44.5, 44.2, 34.5, 28.1, 27.5, 21.3; Minor Diast: 216.2, 137.8, 132.5, 131.4, 130.2, 89.1, 81.3, 45.8, 45.5, 43.0, 35.5, 31.4, 26.8, 22.3; HRMS (EI) calcd for $\mathrm{C}_{17} \mathrm{H}_{20} \mathrm{O}_{2} \mathrm{~S}\left(\mathrm{M}^{+}\right)$288.1184; found 283.1180; Anal. Calcd for $\mathrm{C}_{17} \mathrm{H}_{20} \mathrm{O}_{2} \mathrm{~S}$ : C, 70.80; H, 6.99. Found: C, 70.36; H 7.05.

Isomer 8: m.p. $95{ }^{\circ} \mathrm{C}$; $\mathrm{R}_{f} 0.44$ (3:7 EtOAc/hexanes); IR $\left(\mathrm{CH}_{2} \mathrm{Cl}_{2}\right.$ cast) 2953, 2866, 1711 , 1493, 1448, 1404, 1345, 1301 $\mathrm{cm}^{-1} ;{ }^{1} \mathrm{H}$ NMR (500 MHz, $\left.\mathrm{CDCl}_{3}\right) \square 7.32(\mathrm{~d}, J=8.0 \mathrm{~Hz}$, $2 \mathrm{H}), 7.10(\mathrm{~d}, J=8.0 \mathrm{~Hz}, 2 \mathrm{H}), 4.75(\mathrm{~d}, J=7.3 \mathrm{~Hz}, 1 \mathrm{H}), 3.43\left(\mathrm{~d}, J_{\mathrm{AB}}=14.8 \mathrm{~Hz}, 1 \mathrm{H}\right), 3.32$ $(\mathrm{s}, 1 \mathrm{H}), 2.32(\mathrm{~s}, 3 \mathrm{H}), 2.26-2.21(\mathrm{~m}, 1 \mathrm{H}), 2.24\left(\mathrm{~d}, J_{\mathrm{AB}}=14.8 \mathrm{~Hz}, 1 \mathrm{H}\right), 2.16(\mathrm{~m}, 1 \mathrm{H})$, $2.13(\mathrm{dd}, J=12.4,9.1 \mathrm{~Hz}, 1 \mathrm{H}), 1.93-1.84(\mathrm{~m}, 3 \mathrm{H}), 1.67(\mathrm{~m}, 1 \mathrm{H}), 1.51(\mathrm{ddd}, J=13.4$, 9.8, $7.5 \mathrm{~Hz}, 1 \mathrm{H}), 1.40(\mathrm{~m}, 1 \mathrm{H}) ;{ }^{13} \mathrm{C} \mathrm{NMR}\left(125 \mathrm{MHz}, \mathrm{CDCl}_{3}\right) \square 203.4,138.4,132.9$, 130.1, 93.9, 60.9, 48.9, 45.3, 39.9, 37.1, 34.8, 25.4, 21.4; HRMS (EI) calcd for $\mathrm{C}_{17} \mathrm{H}_{20} \mathrm{O}_{2} \mathrm{~S}\left(\mathrm{M}^{+}\right)$288.1184; found 283.1183; Anal. Calcd for $\mathrm{C}_{17} \mathrm{H}_{20} \mathrm{O}_{2} \mathrm{~S}: \mathrm{C}, 70.80 ; \mathrm{H}$, 6.99. Found: C, 70.90; H, 6.68 . 
Carbene Transfer Reaction of $4 \mathrm{~b} \square$; Preparation of $7 \mathrm{~b} \square$ and $7 \mathrm{~b} \square$. Diazoketone $4 \mathrm{~b} \square$ was subjected to the procedure given above for $4 \mathbf{b} \square$, to yield $85 \%$ of $7 \mathbf{b} \square$ and $7 \mathbf{b} \square$ in a 1:4.8 ratio. None of the isomeric product 8 was isolated.

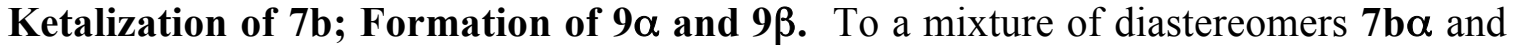
$7 \mathbf{b} \square\left(340 \mathrm{mg}, 1.2 \mathrm{mmol}\right.$, ca. 2.5:1) in $\mathrm{CH}_{2} \mathrm{Cl}_{2}(6 \mathrm{~mL})$ at $0^{\circ} \mathrm{C}$ was added 1,2bis(trimethylsiloxy)ethane $(580 \square \mathrm{L}, 2.4 \mathrm{mmol})$ followed by TMSOTf (12 $\square \mathrm{L}, 5 \mathrm{~mol} \%$ ) and the resulting mixture was warmed to room temperature over several hours. This was then heated to reflux for ca. 12 hours, after which TLC showed consumption of starting materials. The reaction was quenched with pyridine $(1 \mathrm{~mL})$ and saturated aqueous $\mathrm{NaHCO}_{3}(5 \mathrm{~mL})$, diluted with $\mathrm{Et}_{2} \mathrm{O}(10 \mathrm{~mL})$ and the resulting layers separated. The aqueous layer was washed with 3 portions of $\mathrm{Et}_{2} \mathrm{O}(10 \mathrm{~mL})$ and the combined organic extracts were washed with water $(30 \mathrm{~mL})$ then brine $(30 \mathrm{~mL})$, dried over magnesium sulfate, filtered and concentrated, giving the crude product as a yellow oil. This oil was diluted in ether $(20 \mathrm{~mL})$ and passed through a short pad of silica gel in a fritted filter, eluting with ether. This was then concentrated and the resulting oil purified by radial chromatography (silica gel, $2 \mathrm{~mm}$ plate, solvent ramp: $100 \mathrm{~mL}$ each of $3 \%, 6 \%$ then $10 \%$ EtOAc/hexanes until the products are recovered) to yield $9 \square(283 \mathrm{mg}, 72 \%)$ as a pale oil, and $9 \square(102 \mathrm{mg}, 26 \%)$ as a crystalline solid.

Ketal 9匚: $\mathrm{R}_{f} 0.63$ (3:7 EtOAc/hexanes); IR ( $\mathrm{CH}_{2} \mathrm{Cl}_{2}$ cast) 2945, 2869, 1492, 1434, 1398, 1344, 1323, $1291 \mathrm{~cm}^{-1}$; ${ }^{1} \mathrm{H}$ NMR $\left(500 \mathrm{MHz}, \mathrm{CDCl}_{3}\right) \square 7.23(\mathrm{~d}, J=8.0 \mathrm{~Hz}, 2 \mathrm{H}), 7.06(\mathrm{~d}, J$ $=8.0 \mathrm{~Hz}, 2 \mathrm{H}), 4.11(\mathrm{~m}, 1 \mathrm{H}), 4.06(\mathrm{~m}, 1 \mathrm{H}), 3.87,(\mathrm{~d}, J=2.6 \mathrm{~Hz}, 1 \mathrm{H}), 3.84(\mathrm{~m}, 1 \mathrm{H}), 3.76$ $(\mathrm{m}, 1 \mathrm{H}), 3.31(\mathrm{ddd}, J=12.7,5.8,3.5 \mathrm{~Hz}, 1 \mathrm{H}), 2.37\left(\mathrm{~d}, J_{\mathrm{AB}}=13.7 \mathrm{~Hz}, 1 \mathrm{H}\right), 2.42-2.34$ $(\mathrm{m}, 1 \mathrm{H}), 2.32(\mathrm{~s}, 3 \mathrm{H}), 2.10\left(\mathrm{~d}, J_{\mathrm{AB}}=13.7 \mathrm{~Hz}, 1 \mathrm{H}\right), 2.04(\mathrm{dd}, J=14.0,5.7 \mathrm{~Hz}, 1 \mathrm{H}), 1.80-$ $1.74(\mathrm{~m}, 5 \mathrm{H}), 1.65-1.57(\mathrm{~m}, 2 \mathrm{H}) ;{ }^{13} \mathrm{C} \mathrm{NMR}\left(125 \mathrm{MHz}, \mathrm{CDCl}_{3}\right) \square 136.8,134.1,131.1$, 130.3, 116.9, 88.5, 83.1, 65.5, 64.6, 45.7, 45.3, 44.9, 34.6, 28.5, 28.4, 21.4, 21.2; HRMS (EI) calcd for $\mathrm{C}_{19} \mathrm{H}_{24} \mathrm{O}_{3} \mathrm{~S}\left(\mathrm{M}^{+}\right)$332.1146; found 332.1438; Anal. Calcd for $\mathrm{C}_{19} \mathrm{H}_{24} \mathrm{O}_{3} \mathrm{~S}$ : C, 68.64; H, 7.28; S, 9.60. Found: C, 68.78; H, 7.05; S, 9.79.

Ketal 9匚: m.p. $78-79^{\circ} \mathrm{C}$; $\mathrm{R}_{f} 0.54$ (3:7 EtOAc/hexanes); IR $\left(\mathrm{CH}_{2} \mathrm{Cl}_{2}\right.$ cast) 2951, 2871, 1492, 1445, 1433, $1398 \mathrm{~cm}^{-1} ;{ }^{1} \mathrm{H}$ NMR (500 MHz, CDCl $) \square 7.29$ (d, J=8.0 Hz, 2H), $7.05(\mathrm{~d}, J=8.0 \mathrm{~Hz}, 2 \mathrm{H}), 3.92-3.87(\mathrm{~m}, 2 \mathrm{H}), 3.78(\operatorname{app~q}, J=7.2 \mathrm{~Hz}, 1 \mathrm{H}), 3.63$ (ddd, $J=$ 11.5, 7.0, 4.5 Hz, 1H), 3.53 (app q, $J=7.4 \mathrm{~Hz}, 1 \mathrm{H}$ ), 3.38 (ddd, $J=7.8,6.1,1.7 \mathrm{~Hz}, 1 \mathrm{H}$ ), $2.29(\mathrm{~s}, 3 \mathrm{H}), 2.24\left(\mathrm{~d}, J_{\mathrm{AB}}=13.4 \mathrm{~Hz}, 1 \mathrm{H}\right), 2.22(\mathrm{~m}, 1 \mathrm{H}), 1.96\left(\mathrm{~d}, J_{\mathrm{AB}}=13.4 \mathrm{~Hz}, 1 \mathrm{H}\right), 1.92$ - $1.74(\mathrm{~m}, 8 \mathrm{H}) ;{ }^{13} \mathrm{C} \mathrm{NMR}\left(125 \mathrm{MHz}, \mathrm{CDCl}_{3}\right) \square 137.2,132.5,132.4,129.9,116.7,89.0$, 83.4, 65.4, 64.3, 46.1, 44.1 ,43.4, 36.5, 32.1, 27.4, 22.9, 21.3; HRMS (EI) calcd for $\mathrm{C}_{19} \mathrm{H}_{24} \mathrm{O}_{3} \mathrm{~S}\left(\mathrm{M}^{+}\right)$332.1446; found 332.1445; Anal. Calcd for $\mathrm{C}_{19} \mathrm{H}_{24} \mathrm{O}_{3} \mathrm{~S}: \mathrm{C}, 68.64 ; \mathrm{H}$, 7.28; S, 9.60. Found: C, 68.61; H, 7.01; S, 9.76.

LDBB Reduction of 9马; Formation of 10 and 11: A large excess of LDBB was prepared according to Cohen's procedure, using $\mathrm{Li}(8 \mathrm{mg}, 6$ equiv) and di-tertbutylbiphenyl $(300 \mathrm{mg})$ in THF $(3.2 \mathrm{~mL})$. To a solution of $9 \square(57 \mathrm{mg}, 0.19 \mathrm{mmol})$ and a crystal of phenanthroline in THF $(0.7 \mathrm{~mL})$ cooled to $-78^{\circ} \mathrm{C}$ was added $n$-BuLi until the color turned deep red. LDBB was then added dropwise (without quantification) to the reaction mixture until the intense green color persisted, indicating the presence of excess LDBB. The reaction was stirred until consumption of starting material was observed by 
TLC (ca. 2 hours). The reaction was then quenched by addition of saturated aqueous ammonium chloride $(5 \mathrm{~mL})$, further diluted in $\mathrm{Et}_{2} \mathrm{O}(15 \mathrm{~mL})$, and the resulting bi-layer was separated. The aqueous layer was back extracted once with $\mathrm{Et}_{2} \mathrm{O}(10 \mathrm{~mL})$ and the combined organic extracts were pre-dried with brine $(30 \mathrm{~mL})$, dried over anhydrous magnesium sulfate, filtered and concentrated. The crude product was purified by gradient column chromatography (silica gel, $0 \%, 20 \%, 60 \%$ EtOAc/hexanes) to give $\mathbf{1 0}$ (25 mg, $69 \%$ ) as a pale yellow oil, as well as a trace of isomer $11(2 \mathrm{mg}, 5.5 \%)$, in an overall yield of $75 \%$. The ratio of product isomers depended on the time allowed for the reaction: if the reaction was quenched after 30 minutes, the ratio of the products is $\mathbf{1 0}$ (19 $\%)$ and isomer $11(45 \%)$.

Alcohol 10: $\mathrm{R}_{f} 0.36$ (3:7 EtOAc/hexanes); IR ( $\mathrm{CH}_{2} \mathrm{Cl}_{2}$ cast) 3513, 3025, 2959, 1653, $1445,1425,1397,1344,1316,1281 \mathrm{~cm}^{-1}$; ${ }^{1} \mathrm{H}$ NMR $\left(500 \mathrm{MHz}, \mathrm{CDCl}_{3}\right) \square 5.89$ (ddd, $J=$ $12.2,8.9,3.5 \mathrm{~Hz}, 1 \mathrm{H}), 5.65$ (ddd, $J=12.0,3.1,1.3 \mathrm{~Hz}, 1 \mathrm{H}), 4.19(\mathrm{~s}, 1 \mathrm{H}) 3.93(\mathrm{~s}, 4 \mathrm{H})$, 2.29 (dddd, $J=16.1,11.8,3.1,3.1 \mathrm{~Hz}, 1 \mathrm{H}), 2.21\left(\mathrm{~d}, J_{\mathrm{AB}}=14.1 \mathrm{~Hz}, 1 \mathrm{H}\right), 2.04$ (ddd, $J=$ $16.0,8.8,2.3 \mathrm{~Hz}, 1 \mathrm{H}), 1.86\left(\mathrm{~d}, J_{\mathrm{AB}}=14.1 \mathrm{~Hz}, 1 \mathrm{H}\right), 1.80-1.66(\mathrm{~m}, 3 \mathrm{H}), 1.65-1.50(\mathrm{~m}$, $4 \mathrm{H}) ;{ }^{13} \mathrm{C}$ NMR $\left(125 \mathrm{MHz}, \mathrm{CDCl}_{3}\right) \square 133.9,133.6,108.6,78.6,64.6,63.9,47.2,44.9$, 41.9, 30.9, 26.6, 19.8; HRMS (ESI) calcd for $\mathrm{C}_{12} \mathrm{H}_{18} \mathrm{O}_{3} \mathrm{Na}\left(\mathrm{M}^{+}\right)$233.1148; found 233.1149 .

Isomer 11: $\mathrm{R}_{f} 0.09$ (3:7 EtOAc/hexanes); $\mathrm{IR}\left(\mathrm{CH}_{2} \mathrm{Cl}_{2}\right.$ cast) 3428 (br), 2946, 2865, 1651, 1446, 1433, 1360, $1327 \mathrm{~cm}^{-1} ;{ }^{1} \mathrm{H}$ NMR $\left(500 \mathrm{MHz}, \mathrm{CDCl}_{3}\right) \square 5.89(\mathrm{ddd}, J=12.2,8.8,3.5$ $\mathrm{Hz}, 1 \mathrm{H}), 5.64$ (d, $J=12.2 \mathrm{~Hz}, 1 \mathrm{H}), 4.18(\mathrm{~s}, 1 \mathrm{H}), 3.94$ (s, 4H), 2.29 (app ddt, $J=14.5$, $11.3,3.3,3.3 \mathrm{~Hz}, 1 \mathrm{H}), 2.21\left(\mathrm{~d}, J_{\mathrm{AB}}=14.1 \mathrm{~Hz}, 1 \mathrm{H}\right), 2.04(\mathrm{ddd}, J=16.0,8.8,2.2 \mathrm{~Hz}, 1 \mathrm{H})$, $1.86\left(\mathrm{~d}, J_{\mathrm{AB}}=14.1 \mathrm{~Hz}, 1 \mathrm{H}\right), 1.80-1.48(\mathrm{~m}, 7 \mathrm{H}) ;{ }^{13} \mathrm{C} \mathrm{NMR}\left(125 \mathrm{MHz}, \mathrm{CDCl}_{3}\right) \square 153.2$, 99.4, 90.8, 74.1, 67.8, 61.3, 46.0, 46.9, 39.9, 37.1, 33.0, 24.8; HRMS (EI) calcd for $\mathrm{C}_{12} \mathrm{H}_{18} \mathrm{O}_{3}\left(\mathrm{M}^{+}\right)$210.1256; found 210.1253.

Isomer 11 was found to reketalize upon standing in $\mathrm{CDCl}_{3}$ to give compound 12: $\mathrm{R}_{f}$ 0.40 (3:7 EtOAc/hexanes); IR ( $\mathrm{CH}_{2} \mathrm{Cl}_{2}$ cast) $2951,2869,1350 \mathrm{~cm}^{-1} ;{ }^{1} \mathrm{H}$ NMR $(500 \mathrm{MHz}$, $\left.\mathrm{CDCl}_{3}\right) \square 4.43$ (dd, $J=7.6,4.3 \mathrm{~Hz}, 1 \mathrm{H}$ ), 3.92 (app t, $J=6.3 \mathrm{~Hz}, 2 \mathrm{H}$ ), 3.81 (app t, $J=6.3$ $\mathrm{Hz}, 2 \mathrm{H}), 2.67$ (app tt, $J=9.2,4.7 \mathrm{~Hz}, 1 \mathrm{H}), 2.31(\mathrm{dd}, J=12.2,9.0 \mathrm{~Hz}, 1 \mathrm{H}), 2.08\left(\mathrm{~d}, J_{\mathrm{AB}}=\right.$ $13.5 \mathrm{~Hz}, 1 \mathrm{H}), 1.96-1.86(\mathrm{~m}, 2 \mathrm{H}), 1.86-1.78(\mathrm{~m}, 2 \mathrm{H}), 1.72(\mathrm{dd}, J=13.5,2.0 \mathrm{~Hz}, 1 \mathrm{H})$, $1.65-1.61(\mathrm{~m}, 1 \mathrm{H}), 1.64(\mathrm{ddd}, J=13.8,1.8,1.8 \mathrm{~Hz}, 1 \mathrm{H}), 1.56-1.51(\mathrm{~m}, 1 \mathrm{H}), 1.45-$ $1.40(\mathrm{~m}, 1 \mathrm{H}), 1.35-1.30(\mathrm{~m}, 1 \mathrm{H}) ;{ }^{13} \mathrm{C}$ NMR $\left(125 \mathrm{MHz}, \mathrm{CDCl}_{3}\right) \square$ 107.6, 91.5, 76.0, 64.3, 63.2, 44.5, 44.2, 40.7, 39.0, 37.4, 34.9, 25.1; HRMS (EI) calcd for $\mathrm{C}_{12} \mathrm{H}_{18} \mathrm{O}_{3}\left(\mathrm{M}^{+}\right)$ 210.1256 ; found 210.1253 .

i. Diazo compounds and especially diazomethane present special safety hazards. The reader is referred to Aldrich technical bulletin AL-180, Diazald, MNNG and Diazomethane Generators, Aldrich Chemical Co., Milwaukee, WI; available on the internet in pdf format at http://www.sigmaaldrich.com/aldrich/bulletin/AL-180.pdf. 
$89 v \cdot v \tau+$

$\varepsilon 96^{\circ} \varepsilon z^{-2}$

$s 8 \mathrm{~s}^{\circ} \varepsilon \varepsilon$

$\angle I Z \cdot V \varepsilon$

$\mathrm{SS} 8.8 \varepsilon$

EZS.0

D8E. IV

$6 \angle 8^{\circ} \cup V$

$\angle Z 0.9 v-7$

$9 \angle \tau \cdot 9 V$

$\varepsilon 69 . v s$

$s 26^{\circ} b s$

เ $9 \varepsilon^{\circ} 09$

I9D.09

086.92

$900^{\circ} \angle L$

$S \varepsilon z \cdot \ll<\backslash$

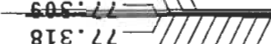

$8 I \varepsilon^{\circ} L L$

$0 D V \cdot L L$

$060^{\circ} \angle L$

$\angle E S^{\circ} \angle L$

$855^{\circ} L L$

$82 S^{\circ} \angle 2$

V 65.22

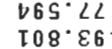

SI 6.26

DZL. $90 \mathrm{I}$

SI0 $\angle 0 T$

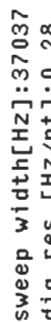

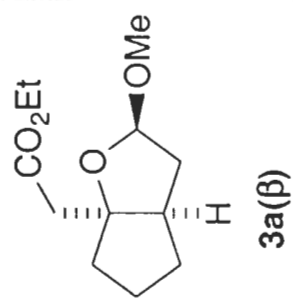

ำ

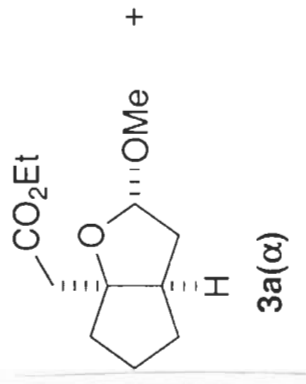

$\angle I Z \cdot I \angle I$

हen 


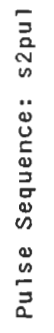
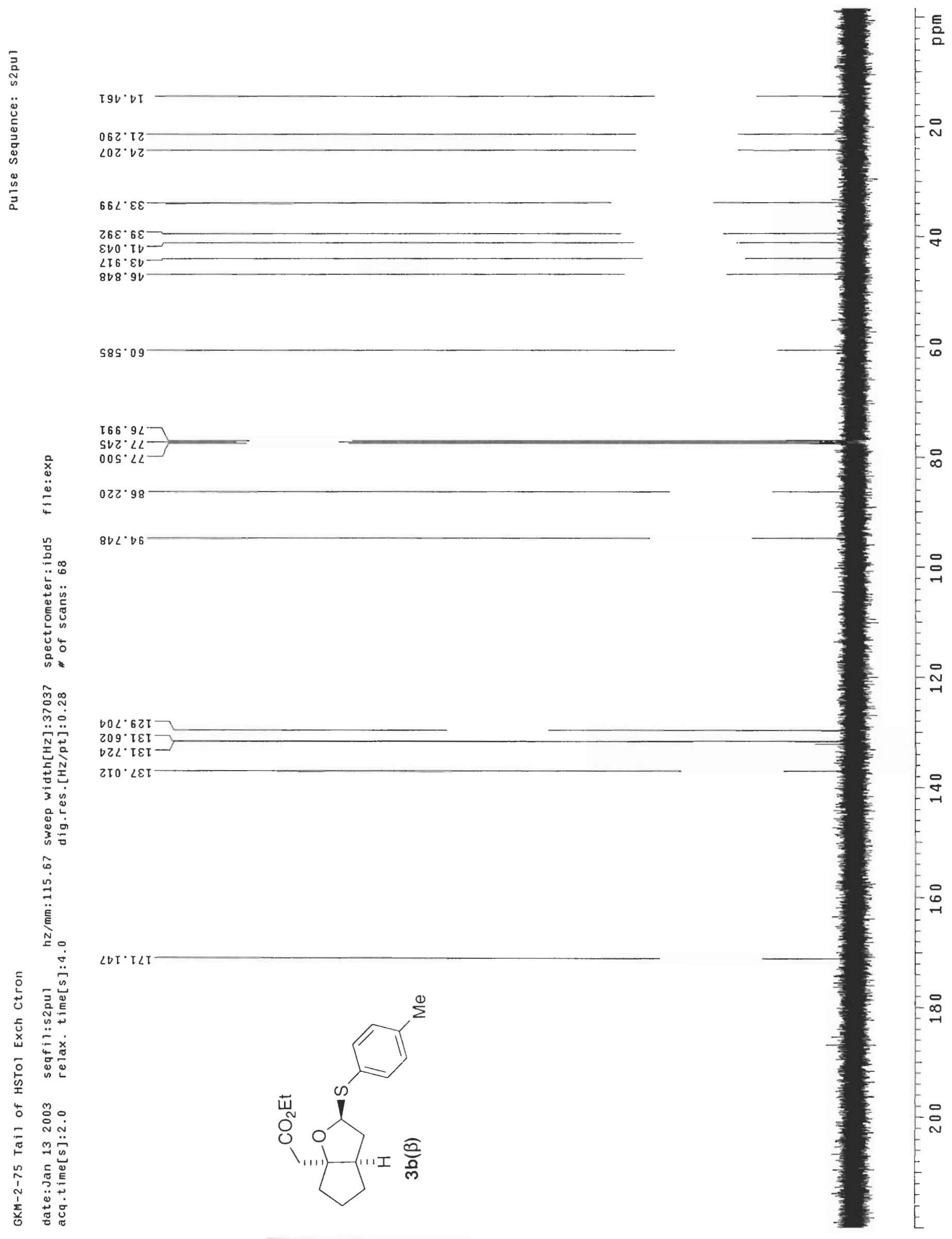

S- 11 


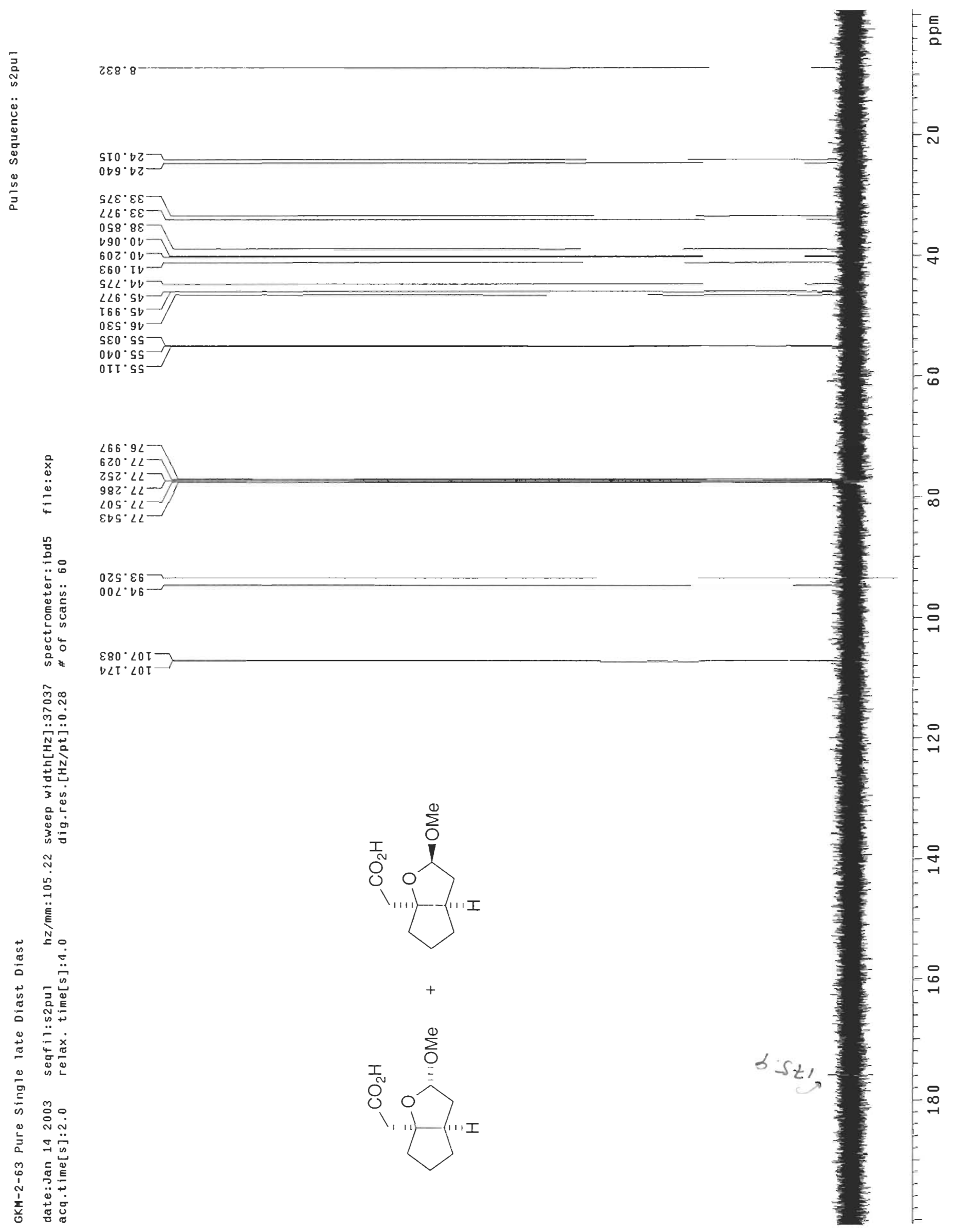

S- 12 


$$
1
$$




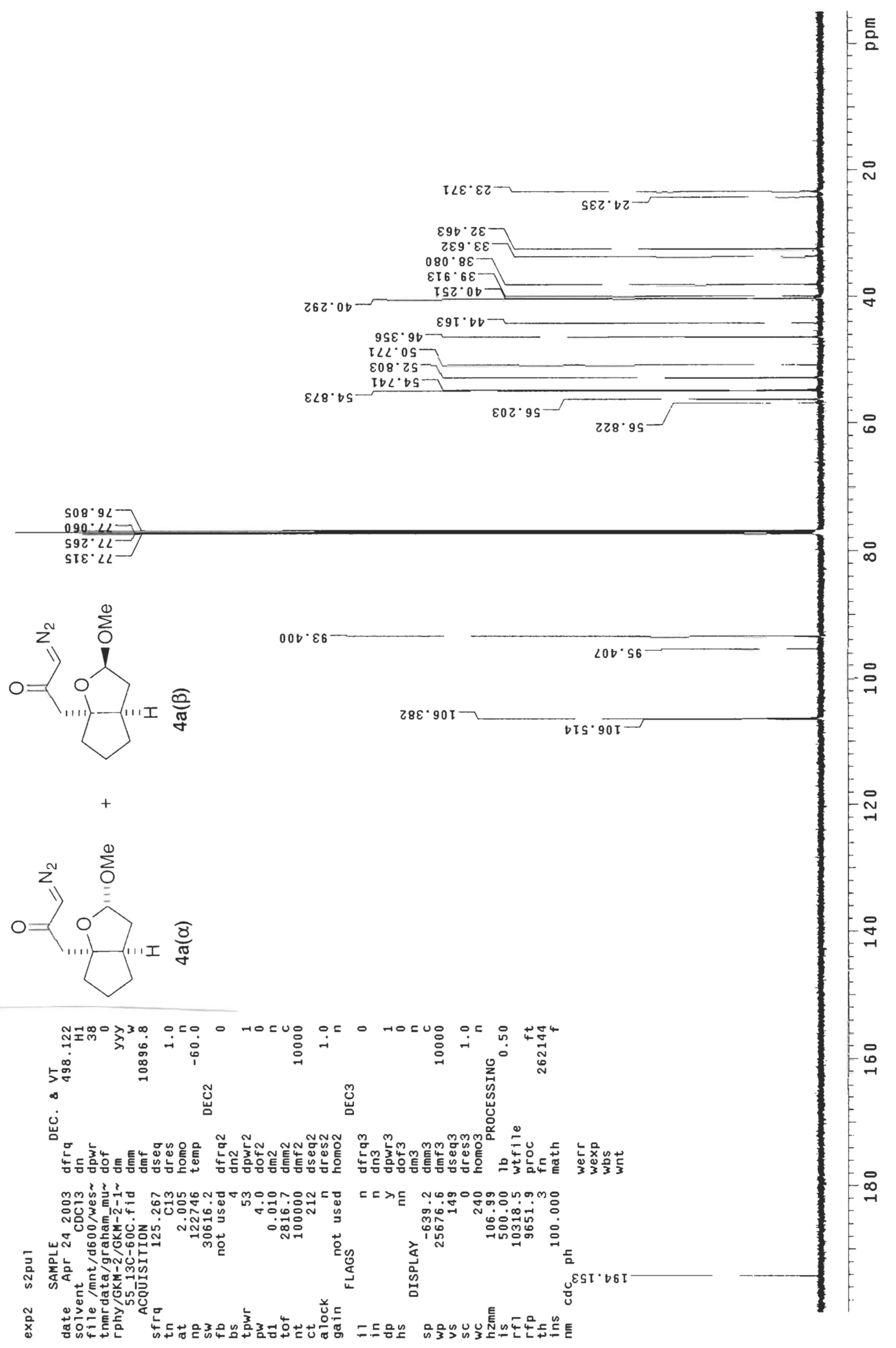

S- 14 
$9 S 6^{\circ} 8 \varepsilon$

$2 S D^{\circ} 00$

$698^{\circ} \circ 0$

$\varepsilon \angle b^{\circ} \angle b$

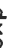

$886^{\circ} 9 L$

$600^{\circ} \angle L$

$\varepsilon 10^{\circ}<L$

$850^{\circ} \angle L$

$=820 \cdot \angle L$

$0<2 \cdot \angle L$

$S \angle Z \cdot \angle L$

$6 \angle 2^{\circ} \angle L$

$082^{\circ}<2$

$860^{\circ} \angle L$

ZES $\angle L S^{\circ} \angle L$

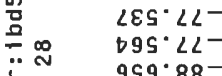

999.88

SS9.v6

$\angle 9 L^{\circ} O E I$

$90 \mathrm{~S}^{\circ} \textrm{ } \varepsilon \mathrm{\tau}$

$6 โ 6^{\circ} \angle \varepsilon \tau$

$\stackrel{n}{-1}$

浐

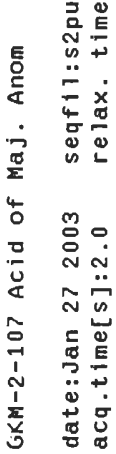

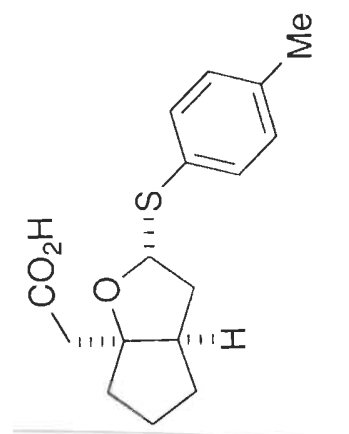

$0 Z 8 \cdot 98 \mathrm{I}$

$228 \cdot 981$
$628 \cdot 981$
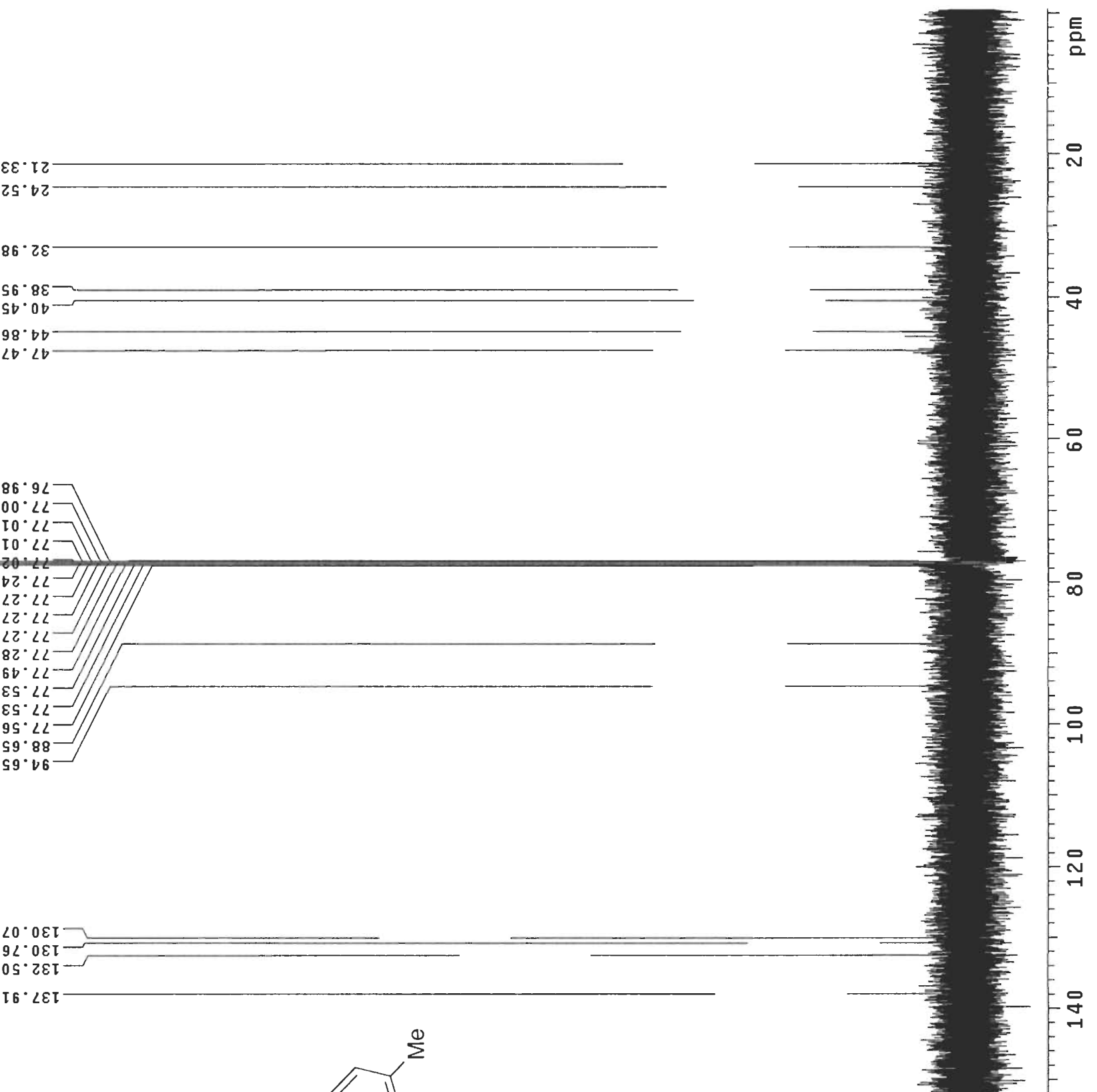
$\tau \angle S^{\circ} \varepsilon \varepsilon-$

$\mathrm{SBS} \cdot 0 \mathrm{~b}-$

$\angle \tau 9^{\circ} \varepsilon b$

$06 \mathrm{~S}^{\circ} \angle \mathrm{b}$

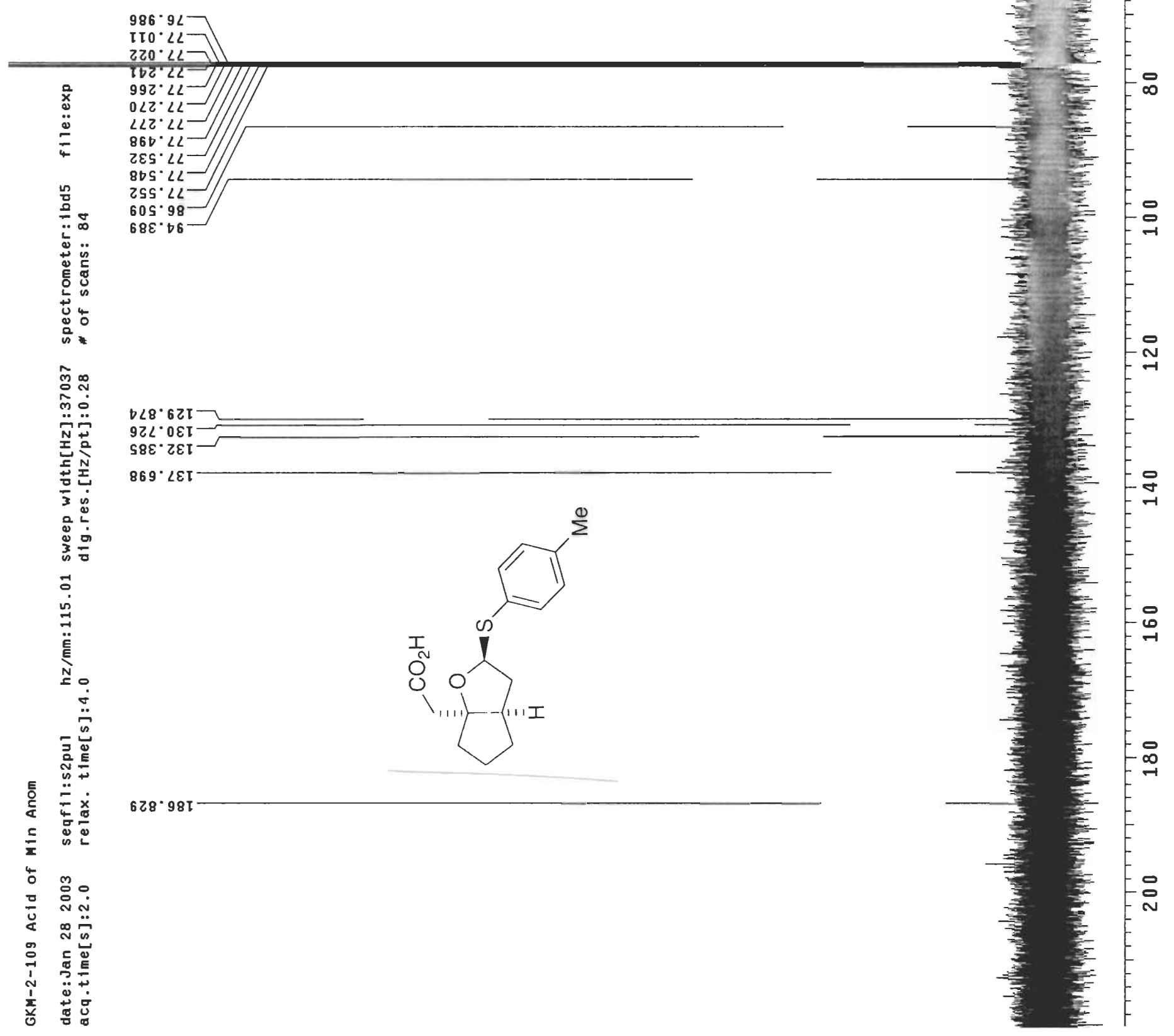

S- 16 


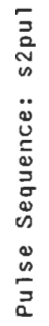
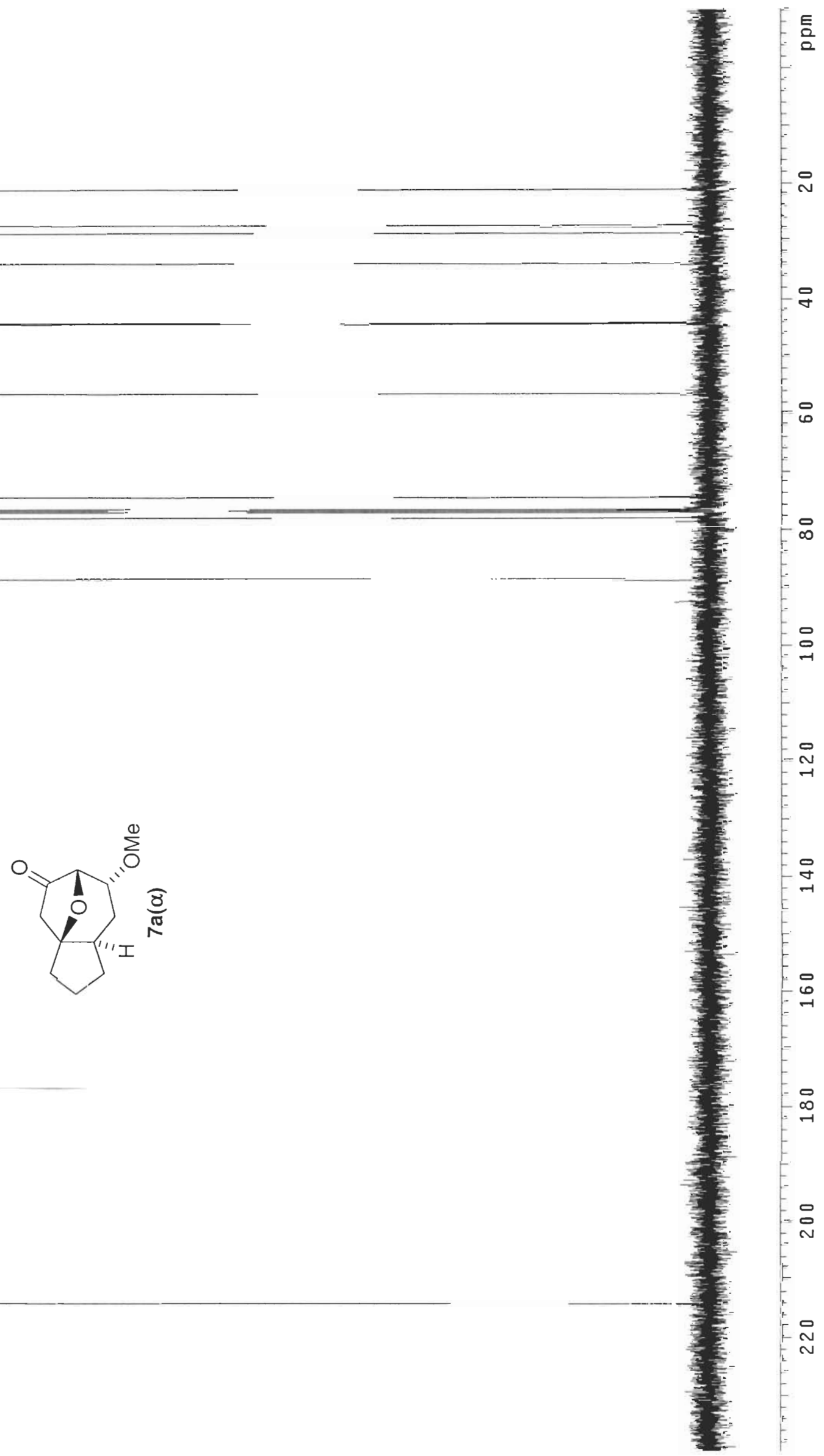

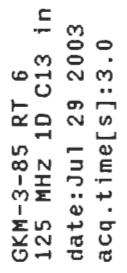

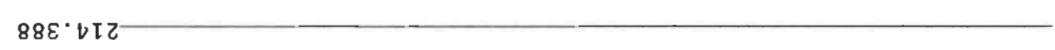

S- 17 


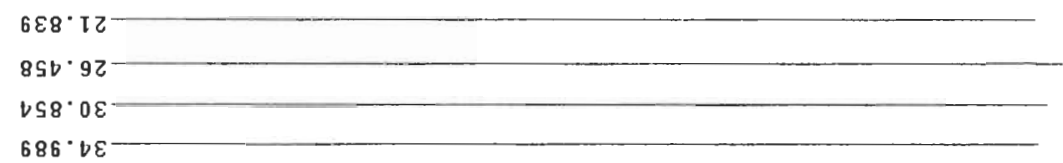

S98. TV

$0 b E^{\circ} s v$

$09 \varepsilon^{\circ} 9 \varsigma$

$58 Z^{\circ} \mathrm{SL}$

$8 \varepsilon L^{\circ} 9 L$

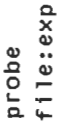

$\varepsilon 66 \cdot 9 L$

$6 V 2 \cdot 12$

$989 \cdot 88$

3

ن

용

స

" Еँ

을 先的

- 幺.

艺呑

१ले

u满芳

ㄴ.

N

量告

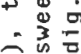

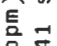

믐

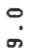

ब

๑

m N

O

$\circ=$

4 竞.

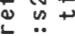

$\Xi \dot{x}$

$\frac{\overline{4}}{4} \frac{x}{0}$

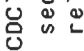

$\therefore$ mo

in $\frac{m}{-1} \stackrel{\circ}{N}$

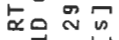

经正离

他 돌

并第要
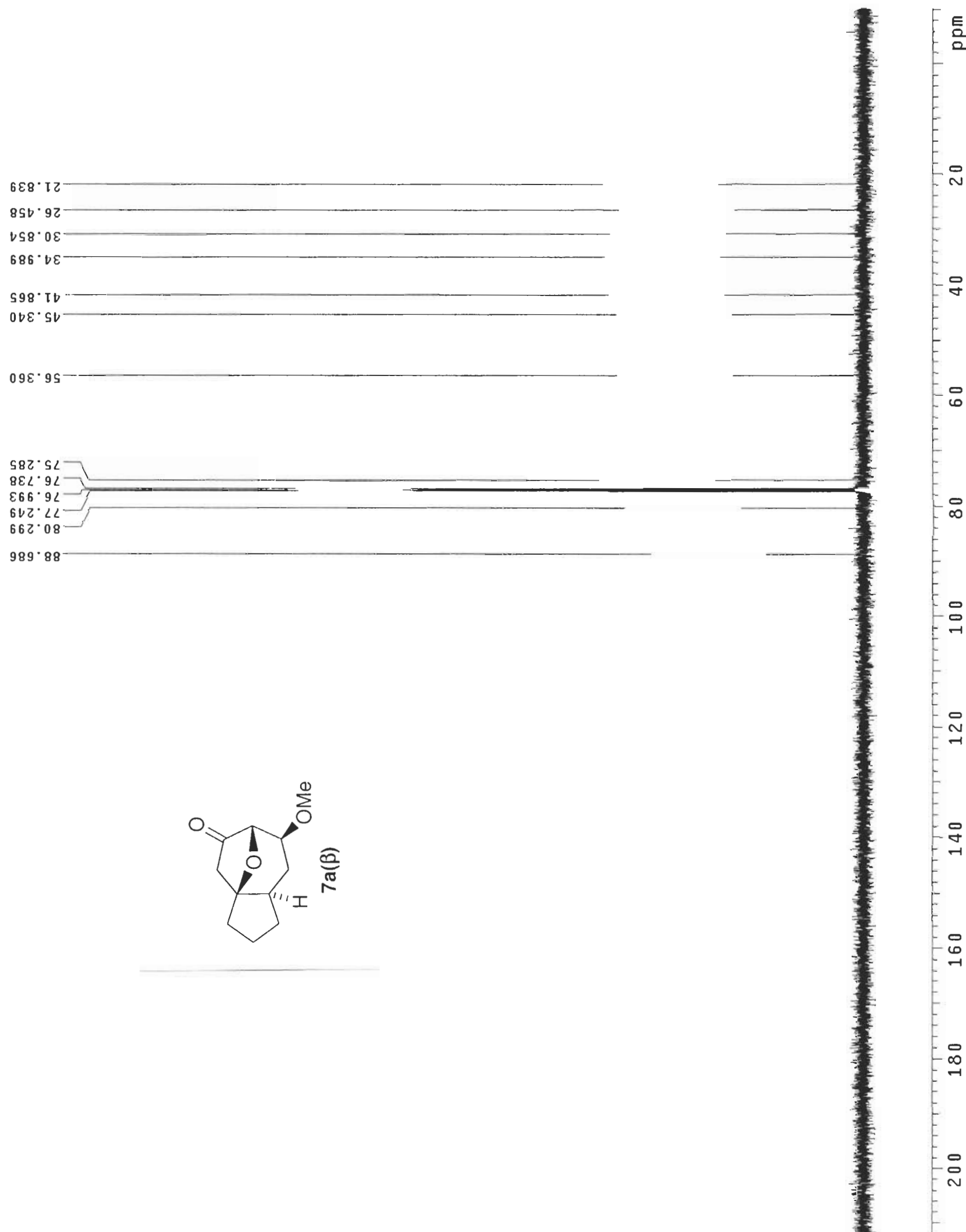

$\angle 28^{\circ} 512$

S- 18 


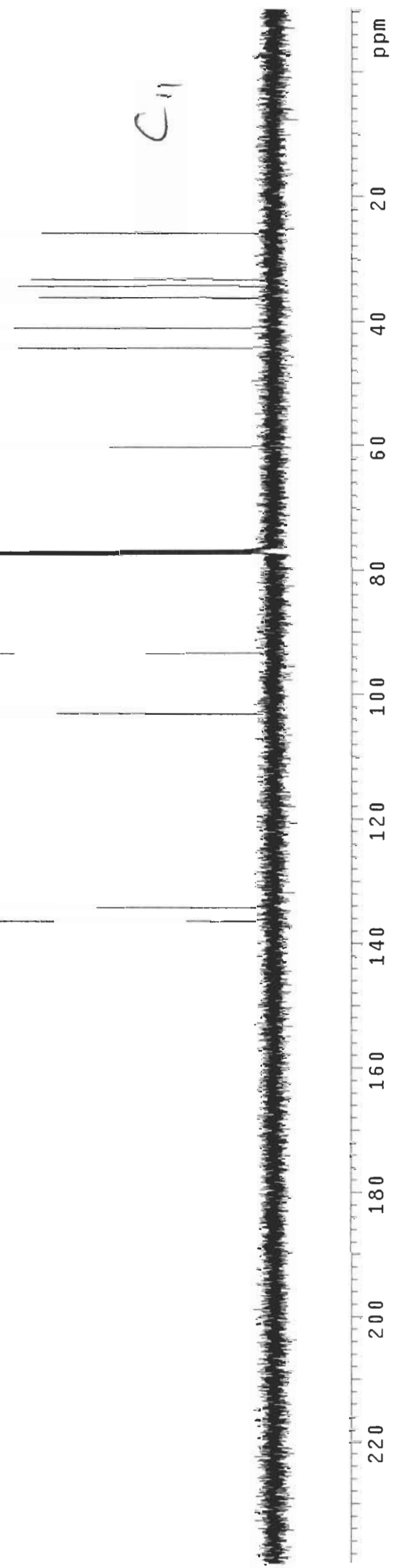

S- 19 
IS $8^{\circ}$ IV

$826 \cdot 00$

$\angle S I \cdot \angle D$

$265 \cdot 92$

$\angle 98^{\circ} \varepsilon 9$

SSS.

$\angle I L \cdot 9 L$

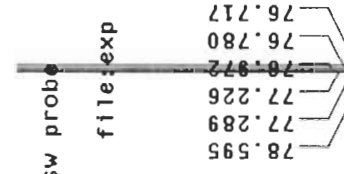

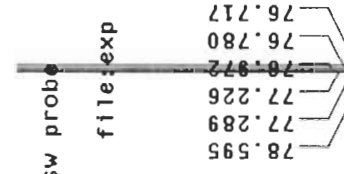

is

i :

$\therefore \quad \ddot{2} \div$

$\stackrel{\sim}{\sim} \stackrel{4}{\stackrel{4}{*}}$

I)

E

$\stackrel{0}{3}$

$6 S S^{\cdot} 80 \mathrm{I}$

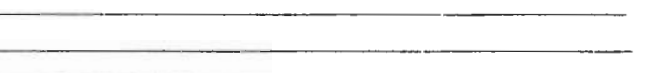

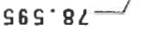

(6)

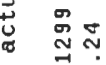

ค

○ N空

ํ.

总

กิ

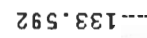

$606 . \varepsilon \varepsilon I$

틍 농

$\therefore$

\%

o

M N

ठั

¿

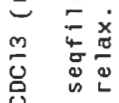

$=9$

m

0 $\sim \ddot{a}$

슬

变

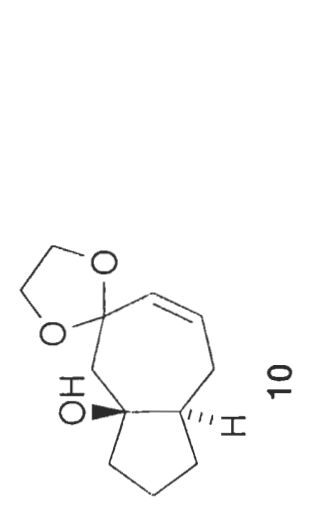

学 


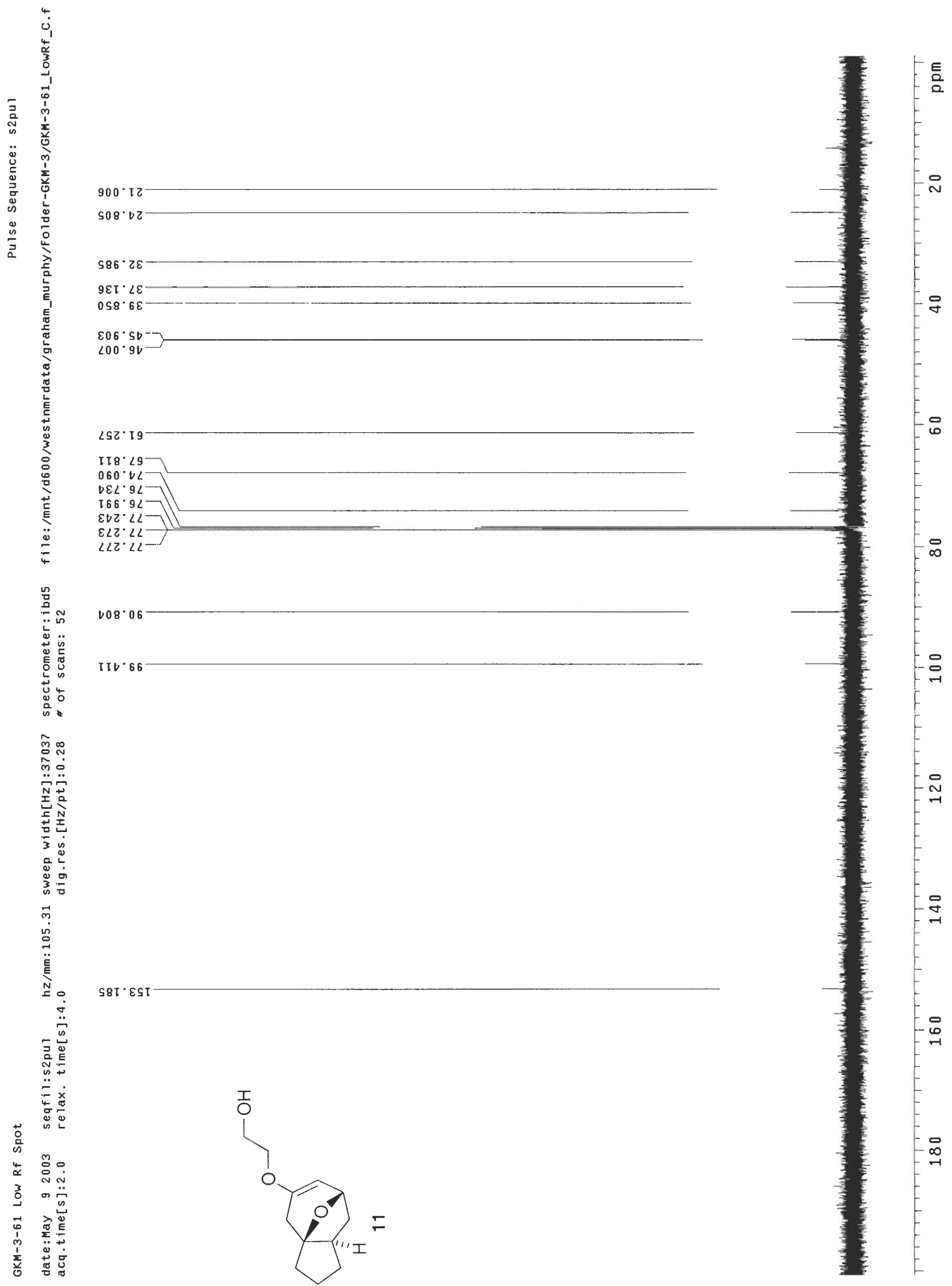

S- 21 

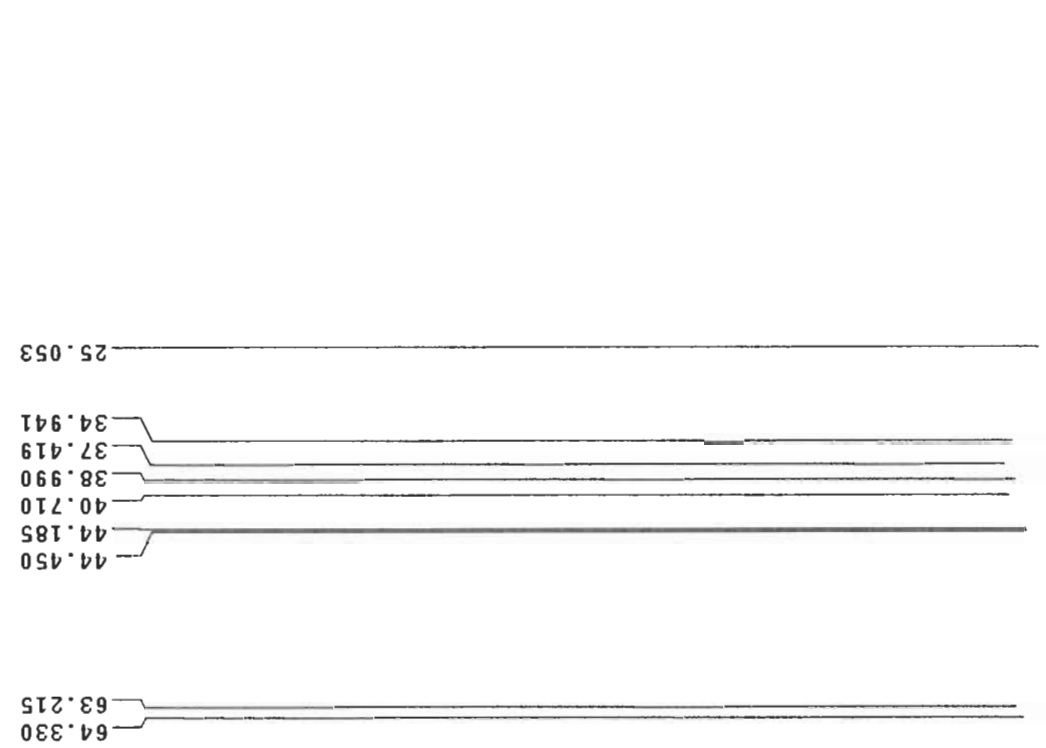

$200 \cdot 9 L$

$S Z L \cdot 9 L$

$\begin{array}{ll} & 0 \\ 0 & 0 \\ 0 & 0 \\ 0 & \frac{0}{0} \\ \frac{0}{2} & \frac{0}{4}\end{array}$

$8 \angle 6^{\circ} 9 L$

$2 \angle 0 \cdot 16$

is

is 号

- $\because \mathrm{m}$

$\approx \stackrel{\circ}{*} \quad 9 \angle s^{\circ} \angle 0 \tau$

11 焉

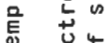

离 40

离

$\stackrel{5}{N} \bar{N}$

१

0 N罙

$\therefore$ 논

ก 논

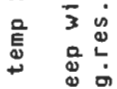

- $\quad \frac{0}{3}$

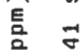

वे

产

రั

+ $\div$

诺

m

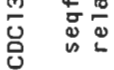

용.

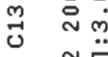

요 $N$

돌

م

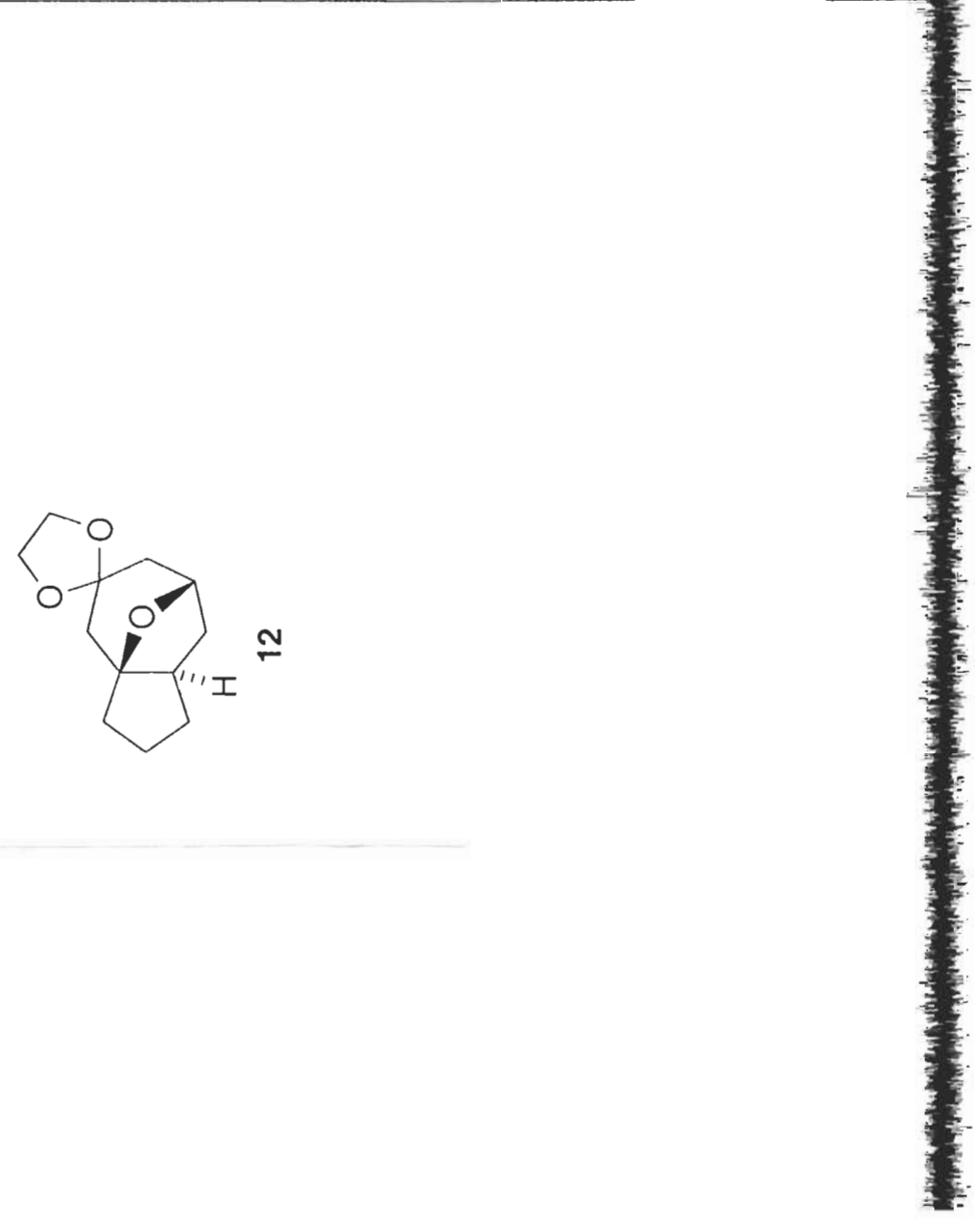

S- 22 